\title{
Sox2 Is Essential for Oligodendroglial Proliferation and Differentiation during Postnatal Brain Myelination and CNS Remyelination
}

\author{
[DSheng Zhang, ${ }^{1,2 *}$ Xiaoqing Zhu, ${ }^{1,2,3 *}$ Xuehong Gui, ${ }^{1}$ Christopher Croteau, ${ }^{1}$ Lanying Song, ${ }^{1,2}$ Jie Xu, ${ }^{1}{ }^{-}$Aijun Wang, ${ }^{1,4}$ \\ Peter Bannerman, ${ }^{1,5}$ and $\odot$ Fuzheng Guo ${ }^{1,2}$ \\ ${ }^{1}$ Institute for Pediatric Regenerative Medicine, Shriners Hospitals for Children, Northern California, California 95817, ${ }^{2}$ Department of Neurology, ${ }^{3}$ Cancer \\ Institute, The Affiliated Hospital of Qingdao University, Qingdao, Shandong, China, ${ }^{4}$ Department of Surgery, and ${ }^{5}$ Department of Cell Biology and Human \\ Anatomy, School of Medicine, University of California-Davis, California 95817
}

In the CNS, myelination and remyelination depend on the successful progression and maturation of oligodendroglial lineage cells, including proliferation and differentiation of oligodendroglial progenitor cells (OPCs). Previous studies have reported that Sox 2 transiently regulates oligodendrocyte $(\mathrm{OL})$ differentiation in the embryonic and perinatal spinal cord and appears dispensable for myelination in the postnatal spinal cord. However, the role of Sox 2 in OL development in the brain has yet to be defined. We now report that Sox 2 is an essential positive regulator of developmental myelination in the postnatal murine brain of both sexes. Stage-specific paradigms of genetic disruption demonstrated that Sox 2 regulated brain myelination by coordinating upstream OPC population supply and downstream OL differentiation. Transcriptomic analyses further supported a crucial role of Sox 2 in brain developmental myelination. Consistently, oligodendroglial Sox2-deficient mice developed severe tremors and ataxia, typical phenotypes indicative of hypomyelination, and displayed severe impairment of motor function and prominent deficits of brain OL differentiation and myelination persisting into the later CNS developmental stages. We also found that Sox2 was required for efficient $\mathrm{OPC}$ proliferation and expansion and OL regeneration during remyelination in the adult brain and spinal cord. Together, our genetic evidence reveals an essential role of Sox 2 in brain myelination and CNS remyelination, and suggests that manipulation of Sox 2 and/or Sox2-mediated downstream pathways may be therapeutic in promoting CNS myelin repair.

Key words: myelination and remyelination; oligodendrocyte differentiation; oligodendrocyte regeneration; oligodendroglial lineage progression; oligodendroglial progenitor cells; Sox2

Significance Statement

Promoting myelin formation and repair has translational significance in treating myelin-related neurological disorders, such as periventricular leukomalacia and multiple sclerosis in which brain developmental myelin formation and myelin repair are severely affected, respectively. In this report, analyses of a series of genetic conditional knock-out systems targeting different oligodendrocyte stages reveal a previously unappreciated role of Sox 2 in coordinating upstream proliferation and downstream differentiation of oligodendroglial lineage cells in the mouse brain during developmental myelination and CNS remyelination. Our study points to the potential of manipulating Sox2 and its downstream pathways to promote oligodendrocyte regeneration and CNS myelin repair.

\section{Introduction}

The transcription factor SRY (sex-determining region)-box 2 (Sox2) is a critical transcription factor in regulating the proper-

Received May 10, 2017; revised Dec. 11, 2017; accepted Jan. 8, 2018.

Author contributions: S.Z., X.Z., and F.G. designed research; S.Z., X.Z., X.G., C.C., L.S., J.X., A.W., P.B., and F.G. performed research; A.W., P.B., and F.G. contributed unpublished reagents/analytic tools; S.Z., X.Z., X.G., C.C., and F.G. analyzed data; S.Z., X.Z., and F.G. wrote the paper.

This work was supported by the National Institutes of Health Grants R01NS094559 and R21NS093559 to F.G., National Institutes of Health Grant R01NS100761 to A.W., and Shriners Hospitals for Children research grants to F.G. and postdoctoral fellowship grant to S.Z. We thank Dr. Q. Richard Lu (University of Cincinnati, Cincinnati) for critical comments on the manuscript; and Daffcar Erol (University of California-Davis) for the editions. ties of stem cells, including neural stem cells (Zhang and Cui, 2014), and it is the key determining factor for in vivo reprogramming of differentiated neural cells into neural precursor cells (Niu et al., 2013; Heinrich et al., 2014). In the CNS, Sox2 was

The authors declare no competing financial interests.

*S.Z. and X.Z. contributed equally to this study as co-first authors.

Correspondence should be addressed to Dr. Fuzheng Guo, Department of Neurology, University of CaliforniaDavis School of Medicine, c/o Shriners Hospitals for Children, 2425 Stockton Blvd, Sacramento, CA 95817. E-mail: fzguo@ucdavis.edu.

D0I:10.1523/JNEUROSCI.1291-17.2018

Copyright $\odot 2018$ the authors $\quad 0270-6474 / 18 / 381802-19 \$ 15.00 / 0$ 
originally thought to inhibit the neuronal differentiation of neural stem/progenitor cells (NSPCs) (Graham et al., 2003). However, later genetic studies demonstrate that Sox2 positively regulates neuronal differentiation from NSPCs (Ferri et al., 2004; Episkopou, 2005).

In oligodendroglial lineage cells, Sox 2 has been reported to be absent from in vitro oligodendroglial progenitor cells (OPCs) (Kondo and Raff, 2004; Lyssiotis et al., 2007). Recent studies demonstrate that in vivo OPCs constantly express low levels of Sox2 (Shen et al., 2008; Dai et al., 2015) and propose that Sox2 maintains OPC proliferation and plays an inhibitory role in oligodendrocyte (OL) differentiation and regeneration (Shen et al., 2008; Pedre et al., 2011). Until recently, genetic evidence suggests that Sox 2 is an essential regulator of OL terminal differentiation, but dispensable for OPC proliferation and migration in the embryonic and perinatal spinal cord (Hoffmann et al., 2014). A subsequent study by Zhao et al. (2015) shows that Sox2 appears dispensable for developmental myelination in the postnatal spinal cord; instead, it has a crucial role in recruiting adult OPCs into the chemical-induced spinal demyelinating lesions during spinal cord myelin repair. These discrepant results strongly suggest that Sox 2 may play a context-dependent role in regulating CNS oligodendrocyte development and regeneration. In this regard, the functions of Sox 2 in brain myelination and remyelination have yet to be defined.

We found that Sox 2 is expressed in all OPCs in the postnatal and adult CNS, and that Sox 2 is transiently upregulated in newly differentiated OLs during developmental myelination and in newly regenerated OLs during remyelination. Using in vivo gene conditional knock-out (cKO), we demonstrate that Sox 2 is essential not only for OPC proliferation and population expansion, but also for downstream OL differentiation during developmental myelination in the murine brain. We also demonstrate that Sox 2 is required for OPC proliferation and OL regeneration after myelin damage in the adult brain and spinal cord. Our study suggests a context-dependent role of Sox 2 in regulating CNS oligodendrocyte development and regeneration.

\section{Materials and Methods}

Transgenic mice. All transgenic mice were maintained on a C57BL/6 background and covered by Institutional Animal Care and Use Committee protocols approved by University of California-Davis. The Cnp-Cre (Lappe-Siefke et al., 2003) (RRID:MGI_3051754) and Rosa-EYFP (RRID:IMSR_JAX:006148) transgenic mice were described in our previous study (Guo et al., 2012; Hammond et al., 2015). Sox10-Cre (RRID: IMSR_JAX:025807), Pdgfra-CreER ${ }^{T 2}$ (RRID:IMSR_JAX:018280), Sox2CreER $^{\text {T2 }}$ (RRID:IMSR_JAX:017593), and Sox2 ${ }^{\mathrm{fl} / \overline{\mathrm{fl}}}$ (RRID:IMSR_JAX: 013093) transgenic mice were purchased from The Jackson Laboratory. Both male and female mice were used in this study. We crossed Cre lines with Sox ${ }^{\mathrm{fl} / \mathrm{fl}}$ mice to generate Sox $2 \mathrm{cKO}$ mice, in which Cre transgenes were maintained as heterozygosity $\left(\mathrm{Cre}^{+/-}\right)$. We used non-Cre Sox $2^{\mathrm{fl} / \mathrm{fl}}$ as Sox2 wild-type (WT) mice or non-Cre control mice. In our study, we referred to the Cnp-Cre, Sox $2^{\mathrm{fl} / \mathrm{fl}}$ mice as Cnp-Sox 2 cKO mice, Sox10-Cre, $S_{o x} 2^{\mathrm{fl} / \mathrm{fl}}$ as Sox10-Sox2 cKO mice, and Pdgfr $\alpha-C r e E R^{T 2}$, Sox $2^{\mathrm{fl} / \mathrm{fl}}$ mice treated with tamoxifen as Pdgfr $\alpha$-Sox 2 cKO mice. In Sox2-CreER ${ }^{\mathrm{T} 2}$ mice, the Cre transgene is homologously knocked in the endogenous locus of Sox2; therefore, Sox2-CreER ${ }^{T 2}$, Sox $2^{\mathrm{fl} /+}$ mice would be Sox 2 cKO mice after tamoxifen injection and were referred to as Sox2-Sox2 cKO mice.

Tamoxifen preparation and administration. Tamoxifen (T5648; SigmaAldrich) was prepared as described in our previous studies (Lang et al., 2013; Hammond et al., 2015). In the experimental designs of developmental myelination, Pdgfr $\alpha$-CreER ${ }^{T 2}$, Sox $2^{\mathrm{fl} / \mathrm{fl}}$ mice and $\operatorname{Sox} 2^{\mathrm{fl} / \mathrm{fl}}$ controls were intraperitoneally injected with tamoxifen at a dose of $100 \mu \mathrm{g} / \mathrm{g}$ body weight at time points indicated in the figures. In the experimental designs of demyelination/remyelination, adult (2-3 months old) $P d g f r \alpha-C r e E R^{T 2}$, So $x 2^{\mathrm{f} / \mathrm{fl}}$ mice and Sox $2^{\mathrm{f} / / \mathrm{fl}}$ controls were intraperitoneally injected with $5 \mathrm{~d}$ course of daily tamoxifen at a dose of $1 \mathrm{mg}$ per day.

BrdU or EdU preparation and administration. BrdU (B5002, Sigma) or EdU (A10044, Thermo Fisher Scientific) was dissolved in 0.9\% sterile saline at a concentration of $10 \mathrm{mg} / \mathrm{ml}$. BrdU or EdU was intraperitoneally injected to Sox $2 \mathrm{cKO}$ and Sox ${ }^{\mathrm{fl} / \mathrm{fl}}$ control littermates at a dose of 100 $\mu \mathrm{g} / \mathrm{g}$ body weight at time points indicated in the figures.

Myelin oligodendrocyte glycoprotein peptide 35-35-induced experimental autoimmune encephalomyelitis (MOG-peptide ${ }_{35-55} E A E$ ) and cuprizone animal models of CNS demyelination. The procedures of MOG-peptide ${ }_{35-55}$ EAE were conducted as described in our previous study (Guo et al., 2012). Complete Freund's adjuvant (CFA)-immunized mice were used as CFA controls for MOG-peptide ${ }_{35-55}$ EAE. In the MOG-peptide ${ }_{35-55}$ EAE animal model, multifocal inflammatory and demyelination lesions predominantly appear in the lumbar segment of spinal cord. Tamoxifen was intraperitoneally injected to Pdgfr $\alpha$-Sox $2 \mathrm{cKO}$ and Sox $2{ }^{\mathrm{f} / \mathrm{fl}}$ mice for 5 consecutive days starting when mice showed a clinical score $\geq 2$ (Guo et al., 2012). The cuprizone-induced demyelination model was conducted according to our published protocols (Hammond et al., 2015). In the cuprizone model, diffused demyelination lesions predominantly occur in the forebrain corpus callosum.

Antibodies and primers. The antibodies used in immunohistochemical staining and Western blotting included the following: Olig2 (AF2418, RRID: AB_2157554, 1:100; R\&D Systems), Olig2 (18953, RRID:AB_494617, 1:100; IBL), NG2 (AB5320, RRID:AB_91789, 1:200; Millipore), PDGFR $\alpha$ (sc-338, RRID:AB_631064, 1:150; Santa Cruz Biotechnology), O4 (MAB345, RRID: AB_94872, 1:200; Millipore), Sox2 (sc-17320, RRID:AB_2286684, 1:500; Santa Cruz Biotechnology), $\beta$-actin (3700, RRID:AB_2242334, 1:1000; Cell Signaling Technology), Sox10 (sc-17342, RRID:AB_2195374, 1:100; Santa Cruz Biotechnology), BrdU (sc-70441, RRID:AB_1119696, 1:100; Santa Cruz Biotechnology), Ki67 (9129, RRID:AB_10989986,1:200; Cell Signaling Technology), EYFP/GFP (06-896, RRID:AB_310288, 1:500; Millipore), TCF712 (2569S, RRID:AB_2199816,1:200; Cell Signaling Technology; sc8632, RRID:AB_2199825, 1:100; Santa Cruz Biotechnology), MBP (NB600717, RRID:AB_2139899, 1:200; Novus), SMI312 (SMI-312R, RRID: AB_2135329, 1:1000, Covance), active caspase-3 (G748A, RRID:AB_430875, 1:200; Promega), pan-oligodendrocyte marker Clone CC1 (OP80, RRID: AB_213434, 1:200; Calbiochem), and APC (sc-896, RRID:AB_2057493, 1:100; Santa Cruz Biotechnology). Our previous study (Lang et al., 2013) shows that the immunostaining patterns of APC and clone CC1 antibodies are different and that APC is transiently expressed in premyelinating oligodendrocytes. Bin et al. (2016) subsequently demonstrated that antibody clone CCl binds Quaking 7, an RNA binding protein that is highly expressed in myelinating oligodendrocytes. All secondary antibodies were DyLight 488- or DyLight549-conjugated $(\mathrm{Fab})_{2}$ fragments (from Jackson ImmunoResearch Laboratories). Brdu, Edu (Click-iT EdU imaging kits, Invitrogen C10339), and TUNEL (Promega, G3250) immunostaining was performed as in previous studies (Guo et al., 2011; Sohn et al., 2012). qPCR primers were from the PrimerBank at (pga.mgh.harvard.edu/primerbank/).

Primary OPC culture and in vitro differentiation, Western blot, immunohistochemistry, and $q R T-P C R$. The procedures of the above-mentioned experiments were performed according to the published protocols in our previous studies (Guo et al., 2012; Lang et al., 2013; Hammond et al., 2015)

RNA sequencing and data analysis. Total RNA was extracted from forebrains of $P d g f r \alpha-C r e E R^{T 2}$, Sox $2^{\mathrm{fl} / \mathrm{fl}}$ mice $(n=3$, tamoxifen injection at $\mathrm{P} 6$ and P7, forebrain harvested at P14) and non-Cre littermate controls $(n=$ 3, tamoxifen injection at P6 and P7, forebrain harvested at P14) using QIAGEN RNeasy for lipid tissues (catalog \#74804) with on-column DNase I digestion. The quality of RNAs was determined by the Bioanalyzer 2100 system (Agilent Technologies). The cDNA library was prepared using the NEBNext Ultra Directional RNA Library Prep Kit (\#E7420) for Illumina, and sequenced on the Illumina HiSeq 4000 sequencing platform. Single-end clean reads were aligned to the reference genome (mouse genome mm10) using TopHat version 2.0.12. Differentially expressed genes were analyzed using DESeq version 1.10.1, and $p<$ 0.05 was considered as differentially expressed genes. Gene ontology analysis of the differentially expressed genes between Pdgfr $\alpha$-Sox $2 \mathrm{cKO}$ 
and non-Cre controls was performed using the National Institutes of Health online tool DAVID (https://david.ncifcrf.gov/). In our RNA-seq results, the number of total clean reads was similar in Pdgfr $\alpha$-Sox $2 \mathrm{cKO}$ $(1.29 \mathrm{E}+08, n=3)$ to those in non-Cre controls $(1.20 \mathrm{E}+08, n=3$, twotailed Student's $t$ test, $p=0.63$ ). Pearson correlation analysis showed that the intragroup and intergroup variations were neglectable and demonstrated by very high correlation coefficient $\left(R^{2}=0.986-0.996\right)$.

Image acquisition and in vivo Sox 2 density quantification. To quantify nuclear Sox2 density in OPCs and newly differentiated OLs, triple immunohistochemical images (Sox2, PDGFR $\alpha$ or NG2 and TCF712) (see Figs. $1 K, 8 D, G)$ projecting $10 \mu \mathrm{m}$ optical thickness were obtained using Nikon Confocal $\mathrm{C} 1$ and imported to the National Institutes of Health ImageJ for subsequent quantification of Sox 2 density. The nuclei of Sox $2^{+} / \mathrm{PDGFR} \alpha^{+}\left(\right.$or NG2 ${ }^{+}$) OPCs and Sox $2^{+} / \mathrm{TCF} 12^{+}$newly differentiated OLs were outlined by using the "freeform" selection tool of ImageJ. The nuclear Sox2 expression level was presented as Corrected Sox2 Fluorescence Density, which was calculated as the value of Integrated Density - (Area of Selected Nucleus $\times$ Mean Fluorescence of Background). The mean fluorescence of background was calculated by averaging at least four nonstaining locations on the same histological sections. At least 60 PDGFR $\alpha(\mathrm{NG} 2)^{+} \mathrm{OPCs}$ or TCF712 ${ }^{+} \mathrm{OLs}$ from 3 animals were quantified in this study.

Motor function assessment. Motor function was tested by measuring accelerating Rotarod retention times. The parameter settings of the accelerating Rotarod in this study: starting speed $=4.0$ rotations per minute $(\mathrm{rpm})$, speed step $=1.3 \mathrm{rpm}$ every $10 \mathrm{~s}$, ending speed $=40 \mathrm{rpm}$. Mice were trained for three $5 \mathrm{~min}$ sessions daily for 2 consecutive days followed by data collection on the third day. The retention time of each mouse was calculated by averaging the retention duration on the Rotarod of 3 trials.

Toluidine blue staining and transmission electron microscopy. Tissue processing for semithin sections of toluidine blue staining and ultrathin sections of transmission electron microscopy was adapted from our previous protocols (Guo et al., 2015; Sohn et al., 2017). In brief, postnatal $28 \mathrm{~d}$ Sox $10-C r e$, So $22^{\mathrm{fl} / \mathrm{fl}}$ and littermate Sox $2^{\mathrm{fl} / \mathrm{fl}}$ control mice were anesthetized with ketamine and xylazine mixture and perfused with $4 \%(\mathrm{w} / \mathrm{v})$ PFA, followed by $3 \%(\mathrm{w} / \mathrm{v})$ glutaraldehyde. Corticospinal tracts at the lumbar segments $(\sim 1-2 \mathrm{~mm}$ thick) were dissected under the stereoscope for further process. Dissected specimens containing corticospinal tracts were washed with $0.2 \mathrm{M}$ sodium cacodylate buffer, $\mathrm{pH} 7.2$, postfixed in $2 \%(\mathrm{w} / \mathrm{v})$ aqueous osmium tetroxide for $2 \mathrm{~h}$, and dehydrated through ascending alcohols, followed by washing in propylene oxide. The resulting specimens were embedded in EMBed-812 resin. Semithin $(500 \mathrm{~nm})$ sections were cut on a Leica EM UC6 microtome and subjected for toluidine blue staining. Ultrathin $(70-80 \mathrm{~nm})$ sections were cut on a Leica EM UC7 microtome and collected on $12 \mathrm{~mm}$ Formvar-coated copper slot grids, double stained with uranyl acetate and lead citrate, followed by imaging on a CM120 electron microscope.

Experimental design and statistical analyses. For immunohistochemical quantification, $10 \mu \mathrm{m}$ optical thickness sections were obtained by confocal $z$-stacking and projected into one flattened image. The images were from the same anatomic locations of Sox $2 \mathrm{CKO}$ and control mice, and at least three histological sections were analyzed in each animal. In the MOG-peptide EAE experimental designs, due to the stochastic distribution of inflammatory infiltrations in the spinal white matter, we quantified marker-positive cells in the whole coronal sections of lumbar spinal cord, rather than the same anatomic locations. All the counting and calculation were performed using the confocal EZ-C1 viewer application (Nikon). For qRT-PCR quantification, the mRNA expression level of interested genes in each sample was normalized to the internal control, housekeeping gene Hsp90. The equation of $2^{\wedge \text { (Ct of Hsp90 - Ct of interested gene) }}$ was used for gene expression calculation. We used National Institutes of Health ImageJ to quantify protein expression levels by analyzing the scanned grayscale films. The level of protein of interest was normalized to the internal control $\beta$-actin. Two-group comparisons were analyzed by two-tailed Student's $t$ tests. Data were presented as mean \pm SD. A $p$ value $<0.05$ was considered as significant difference. Multiple comparisons were performed with one-way ANOVA, followed by Tukey's post hoc test to determine which two groups were significantly different. A $p$ value $<0.05$ was considered as significant difference. For detailed information of statistical analyses, including sample size, $p$ value, $t$ value, and degrees of freedom, see Table 1 .

\section{Results}

Sox 2 is expressed in OPCs and transiently upregulated in newly differentiated, premyelinating OLs during oligodendroglial lineage progression and maturation

In the corpus callosum of neonatal pups of postnatal day 0 (P0) where no differentiated OLs appear yet, Sox 2 was observed in PDGFR $\alpha^{+}$OPCs (Fig. 1A, arrowheads) and brain lipid basic protein-positive astrocyte precursor cells (Fig. $1 A$, arrows). The observation of Sox 2 expression in astroglial lineage cells was consistent with previous data (Guo et al., 2011; Zhao et al., 2015) and was beyond the scope of the current study. A previous study reported that Sox 2 was expressed in early postnatal OPCs and absent from adult OPCs (Zhao et al., 2015). Similarly, our immunohistochemical data confirmed that early postnatal OPCs in the brain on P5 and P14 expressed Sox2 (Fig. 1B, arrowheads). We found that OPCs in the adult brain retained Sox 2 expression (Fig. $1 C$, arrowheads, left). The expression of Sox 2 in adult OPCs was unequivocally demonstrated by the absence of immunoreactive signals in the tamoxifen-induced Sox $2 \mathrm{cKO}$ brain of adult Pdgfra$\mathrm{CreER}^{T 2}$, So $2^{\mathrm{fl} / \mathrm{fl}}$ mice (Fig. $1 \mathrm{~B}$, arrows, right). Interestingly, Sox 2 was also expressed in differentiated $\mathrm{CCl}^{+}$OLs in the forebrain on P14 (Fig. 1D, left, arrowheads) and spinal cord on P10 (Fig. $1 D$, right, arrowheads), two representative time windows of active myelination in the murine CNS. The proportion of $\mathrm{CC}^{+}$ OLs that were Sox2-positive in the corpus callosum progressively decreased during postnatal brain development $(81.5 \pm 7.0 \%$ at $\mathrm{P} 7,38.7 \pm 5.8 \%$ at $\mathrm{P} 13,4.9 \pm 1.7 \%$ at $\mathrm{P} 60$, mean $\pm \mathrm{SD})$. Particularly, most myelin $\mathrm{MBP}^{+}$(Fig. $1 E$, left) and $\mathrm{CNP}^{+}$(Fig. $1 E$, right) OLs expressed Sox 2 in the forebrain on $\mathrm{P} 7$, a proximate time point that OL differentiation starts in the murine brain.

Using TCF712 to mark newly differentiated OLs (Hammond et al., 2015), we found that Sox2-expressing OLs (Sox $2^{+} / \mathrm{CC}^{+}$) were TCF712-positive in the forebrain (Fig. $1 F$, arrowheads) and spinal cord (Fig. 1G, arrowheads). Quantification data showed that $>90 \%$ of $\mathrm{TCF} 2^{+}$newly differentiated OLs were Sox $2-$ positive in the corpus callosum regardless of the time points we assessed (P7, P10, P14, and P60), although the density of TCF712 ${ }^{+}$ newly differentiated OLs was decreased over time during developmental myelination, reflecting the gradually diminished rate of oligodendrocyte generation (Hammond et al., 2015).

We found that Sox 2 peaks at the onset of OL differentiation. The Sox 2 mRNA level in primary differentiating OLs was significantly increased compared with that in purified primary OPCs that had been isolated from neonatal mouse forebrains (Fig. $1 \mathrm{H}$ ). The Sox2 protein level was also increased in differentiating OLs after OPCs were cultured in the differentiation medium for $1 \mathrm{~d}$ (Fig. 1I). The primary OPC culture and differentiation system were validated by the sharp increases in the expression levels of MBP mRNA (Fig. 1J) and protein (Fig. 1I) in differentiating OLs (MBP mRNA, 700-fold higher in D1 OLs vs OPCs, 20,000fold higher in D2 OLs vs OPCs, and $\sim 80,000$-fold higher in D4 OLs vs OPCs). Particularly, TCF712 was overlapped with Sox2 protein expression in differentiating OLs at D1 and D2 (Fig. 1I), consistent with the immunohistochemical observations (Fig. $1 F, G)$. In line with the in vitro data, triple immunohistochemistry of Sox2, TCF712, and PDGFR $\alpha$ (Fig. $1 K$ ) showed that the corrected Sox2 fluorescence density was $\sim 4$-fold higher in $\mathrm{TCF}_{12} 2^{+}$newly differentiated OLs than that in PDGFR $\alpha^{+}$OPCs in the subcortical white matter tracts of P8 and P14 mice (Fig. $1 L$ ). Collectively, our data demonstrate that Sox 2 is expressed in 
Table 1. Detailed information of statistical analyses

\begin{tabular}{|c|c|c|}
\hline Figures & Sample size $(n)$ & Statistical methods \\
\hline $1 G$ & $n=3$ independent experiments at each time point & $\begin{array}{l}\text { One-way ANOVA ( } p= \\
\text { with Tukey's post }\end{array}$ \\
\hline $1 K$ & $\begin{array}{l}n=1450 \mathrm{OPCs} \text { and } 1160 \mathrm{LL} \text { from } 3 \text { mice at P8 } \\
n=1250 \mathrm{PCs} \text { and } 1010 \mathrm{Ls} \text { from } 3 \text { mice at P14 }\end{array}$ & $\begin{array}{l}\text { Two-tailed unpaired } \\
\text { Student's } t \text { test }\end{array}$ \\
\hline $2 D$ & 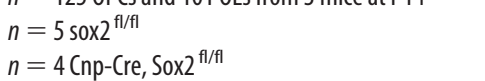 & $\begin{array}{l}\text { Two-tailed unpaired } \\
\text { Student's } t \text { test }\end{array}$ \\
\hline 30 & $\begin{array}{l}n=6 \text { Sox } 2^{\mathrm{fl} / \mathrm{fl}} \\
n=4 \mathrm{Cnp}-\mathrm{Cre}, \mathrm{Sox} \mathrm{2}^{\mathrm{fl} / \mathrm{fl}}\end{array}$ & $\begin{array}{l}\text { Two-tailed unpaired } \\
\text { Student's t test }\end{array}$ \\
\hline $3 E$ & $\begin{array}{l}n=6 \mathrm{sox} 2^{\mathrm{f} / f \mathrm{fl}} \\
n=4 \text { Cnp-Cre, Sox } 2^{\mathrm{fl/fl}}\end{array}$ & $\begin{array}{l}\text { Two-tailed unpaired } \\
\text { Student's t test }\end{array}$ \\
\hline $4 B$ & $\begin{array}{l}n=6 \text { Sox } 2^{f / f f l} \\
n=4 \text { Cnp-Cre, Sox2 } 2^{f / f l}\end{array}$ & $\begin{array}{l}\text { Two-tailed unpaired } \\
\text { Student's } t \text { test }\end{array}$ \\
\hline $4 D$ & $\begin{array}{l}n=8 \text { Sox }{ }^{\mathrm{fl} / \mathrm{fl}} \\
n=4 \text { Cnp-Cre, Sox } 2^{\mathrm{fl} / \mathrm{fl}}\end{array}$ & $\begin{array}{l}\text { Two-tailed unpaired } \\
\text { Student's } t \text { test }\end{array}$ \\
\hline
\end{tabular}

\begin{tabular}{|c|c|}
\hline $4 E$ & $n=3 \mathrm{Sox} 2^{\mathrm{fl} / \mathrm{fl}}$ \\
\hline & $n=4$ Cnp-Cre, Sox $2^{\mathrm{fl} / \mathrm{fl}}$ \\
\hline $5 C$ & $n=7 \mathrm{~S} 0 \times 2^{\mathrm{f} / \mathrm{fl}}$ \\
\hline (cell \#) & $n=7$ Pdgfra-CreER ${ }^{T 2}$, Sox2 $2^{\mathrm{fl} / f 1}$ \\
\hline 50 & $n=4$ Sox $2^{f 1 / f l}$ \\
\hline nRNA) & $n=4$ Pdgfra-CreER ${ }^{\mathrm{T} 2}, \mathrm{Sox} 2^{\mathrm{fl} / \mathrm{fl}}$ \\
\hline $5 F$ & $n=3 \mathrm{Sox} 2^{\mathrm{fl} / \mathrm{fl}}$ \\
\hline & $n=3$ Pdgfra-CreER ${ }^{\mathrm{T} 2}, \mathrm{Sox} 2^{\mathrm{f} / / \mathrm{fl}}$ \\
\hline & $n=6 \mathrm{Sox} 2^{\mathrm{fl} / \mathrm{fl}}$ \\
\hline & $n=6$ Pdgfra-CreER ${ }^{\mathrm{T} 2}, \mathrm{Sox} 2^{\mathrm{f} / \mathrm{fl}}$ \\
\hline
\end{tabular}

Two-tailed unpaired Student's $t$ test Two-tailed unpaired Student's $t$ test Two-tailed unpaired Student's $t$ test Two-tailed unpaired Student's $t$ test

Two-tailed unpaired Student's $t$ test

\author{
Two-tailed unpaired \\ Student's $t$ test \\ Two-tailed unpaired \\ Student's $t$ test \\ Two-tailed unpaired \\ Student's $t$ test \\ Two-tailed unpaired \\ Student's $t$ test \\ Two-tailed unpaired \\ Student's $t$ test \\ Two-tailed unpaired \\ Student's $t$ test \\ Two-tailed unpaired \\ Student's $t$ test
}

Two-tailed unpaired

Student's $t$ test

OPCs: one-way ANOVA $\left(* * * p<0.0001, F_{(2,13)}=24.87\right)$ with Tukey's post test

OLs: one-way ANOVA $\left({ }^{* * *} p=0.0003, F_{(2,13)}=15.84\right)$ with Tukey's post test $p$ value, $t$ value, and $d f$

OPCs versus D1 $0 \mathrm{Ls},{ }^{* *} p<0.01$

OPCs versus D2 $0 \mathrm{Ls},{ }^{*} p<0.05$

OPCs versus $D 40 \mathrm{Ls}, p>0.05$

$\mathrm{P} 8,{ }^{* * *} p<0.0001(t=34.33, \mathrm{df}=259)$

$\mathrm{P} 14,{ }^{* * *} p<0.0001(t=14.87, \mathrm{df}=124)$

$\mathrm{MBP},{ }^{* *} p<0.002(t=4.779, \mathrm{df}=7)$

$\mathrm{SMI} 312, p=0.178(t=1.497, \mathrm{df}=7)$

0 lig2 ${ }^{+} \mathrm{CC} 1^{+},{ }^{* *} p=0.0012(t=4.879, \mathrm{df}=8)$

Olig2 ${ }^{+}$PDGFRa ${ }^{+}, p=0.2314(t=1.295, \mathrm{df}=8)$

$p=0.0041(t=3.975, \mathrm{df}=8)$

0 lig2 ${ }^{+} \mathrm{CC} 1^{+}, p=0.3783(t=0.9326, \mathrm{df}=8)$

Sox2, ${ }^{* *} p=0.0055(t=3.5424, \mathrm{df}=10)$

Mbp, $p=0.5097(t=0.6837, \mathrm{df}=10)$

Plp, $p=0.119(t=1.705, \mathrm{df}=10)$

Mag, $p=0.6681(t=0.4417, \mathrm{df}=10)$

Mobp, $p=0.3381(t=1.006, \mathrm{df}=10)$

Sox10, $p=0.3774(t=0.9237, \mathrm{df}=10)$

$p<0.0001(t=42.99, \mathrm{df}=5)$

Sox $10{ }^{+}$PDGFR $\alpha^{+},{ }^{* * *} p<0.0001(t=6.838, \mathrm{df}=12)$

Sox $10^{+},{ }^{* * * *} p<0.0001(t=7.854, \mathrm{df}=12)$

Sox2, $p=0.0145(t=3.398, \mathrm{df}=6)$

Pdgfra, $p=0.0032(t=4.745, \mathrm{df}=6)$

Sox10 ${ }^{+}, p=0.0009(t=8.856, \mathrm{df}=4)$

Sox $10^{+}$PDGFR $\alpha^{+}, p=0.0007(t=8.856, \mathrm{df}=4)$

Sox $10^{+} \mathrm{CC}^{+}{ }^{+}, p=0.0122(t=4.344, \mathrm{df}=4)$

Sox2, $p=0.0042(t=3, \mathrm{df}=10)$

Plp-Exon3b, $p=0.0027(t=3.964, \mathrm{df}=10)$

Mbp, $p=0.0002(t=5.638, \mathrm{df}=10)$

Mog, $p=0.0272(t=2.585, \mathrm{df}=10)$

Bcas1, ${ }^{* * *} p<0.0001(t=10.02, \mathrm{df}=10)$

Ugt8a, ${ }^{* * *} p<0.0001(t=8.683, \mathrm{df}=10)$

Bmp4, ${ }^{* * *} p<0.0001(t=11.27, \mathrm{df}=10)$

$Q \mathrm{k}, p=0.0012(t=4.461, \mathrm{df}=10)$

${ }^{* * *} p<0.0001(t=7.504, \mathrm{df}=12)$

$\mathrm{EdU}^{+}$Sox $10^{+}$cells, $p=0.0137(t=3.053, \mathrm{dt}=9)$

$\mathrm{Ki}_{67}{ }^{+}$Sox $10^{+}$cells, $^{* * *} \mathrm{p}<0.0001(t=7.544, \mathrm{df}=7)$

Caspase ${ }^{+}$Sox10 ${ }^{+}, p=0.4683(t=0.8008, \mathrm{df}=4)$

TUNEL ${ }^{+}$Sox $10^{+}, p=0.1737(t=1.653, \mathrm{df}=4)$

${ }^{* * *} p<0.0001(t=7.163, \mathrm{df}=10)$

MBP, $p=0.0059(t=3.904, \mathrm{df}=7)$

SMI312, $p=0.6733(t=0.4398, \mathrm{df}=7)$

Sox2, ${ }^{* * *} p<0.0001(t=18.73, \mathrm{df}=5)$

Sox3, $p=0.9522(t=0.06302, \mathrm{df}=5)$

MBP, $p=0.0098(t=4.052, \mathrm{df}=5)$

CNP, $p=0.0072(t=4.378, \mathrm{df}=5)$

MAG, $p=0.0278(t=3.070, \mathrm{df}=5)$

Sox2, $p=0.0116(t=3.157, \mathrm{df}=9)$

Mbp, $p=0.0093(t=3.298, \mathrm{df}=9)$

Plp-E3b, $p=0.0404(t=2.392, \mathrm{df}=9)$

Sox2fl/fl versus Sox10-Cre, Sox2fl/,$+ p>0.05$

Sox2fl/fl versus Sox10-Cre, Sox2fl/fl, ${ }^{* * *} p<0.0001$

Sox10-Cre, Sox2fl/ + versus Sox10-Cre, Sox2fl/fl, ${ }^{* * *} p<0.001$

Sox2fl/fl versus Sox10-Cre, Sox2fl/,$+ p>0.05$;

Sox2fl/fl versus Sox10-Cre, Sox2fl/fl, ${ }^{* * *} p<0.0001$

Sox10-Cre, Sox2fl/ + versus Sox10-Cre, Sox2fl/fl, ${ }^{* *} p<0.01$ 
Table 1. Continued

\begin{tabular}{|c|c|c|c|}
\hline Figures & Sample size $(n)$ & Statistical methods & $p$ value, $t$ value, and df \\
\hline 71 & $n=6 \mathrm{Sox} 2^{\mathrm{fl} / \mathrm{fl}}$ & Two-tailed unpaired & PDGFR $\alpha^{+}$OPCs, ${ }^{* * *} p<0.0001(t=6.392, \mathrm{df}=8)$ \\
\hline & $n=4$ Sox $10-$ Cre, Sox $2^{f / f l}$ & Student's $t$ test & $\mathrm{CC}^{+} 0 \mathrm{Ls},{ }^{* *} p<0.001(t=4.848, \mathrm{df}=8)$ \\
\hline $8 D$ & $n=890 \mathrm{OCs}$ and $800 \mathrm{Ls}$ from 3 mice & Two-tailed unpaired Student's $t$ test & ${ }^{* * *} p<0.0001(t=25.89, \mathrm{df}=167)$ \\
\hline 86 & $\begin{array}{l}n=4 \mathrm{CFA} \\
n=4 \mathrm{MOG}\end{array}$ & $\begin{array}{l}\text { Two-tailed unpaired } \\
\text { Student's } t \text { test }\end{array}$ & ${ }^{* * *} p<0.0001(t=17.9, \mathrm{df}=6)$ \\
\hline 81 & $n=990 \mathrm{PCs}$ and $62 \mathrm{OLs}$ from 3 mice & $\begin{array}{l}\text { Two-tailed unpaired } \\
\text { Student's } t \text { test }\end{array}$ & ${ }^{* *} p<0.0001(t=20.67, \mathrm{df}=159)$ \\
\hline $9 B 3$ & $\begin{array}{l}n=3 \mathrm{Sox} 2^{\mathrm{fl} / \mathrm{fl}} \\
n=3 \text { Pdgfra-CreER }{ }^{\mathrm{T} 2}, \mathrm{~S}_{0 \times 2^{\mathrm{fl} / \mathrm{fl}}}\end{array}$ & $\begin{array}{l}\text { Two-tailed unpaired } \\
\text { Student's t test }\end{array}$ & Sox2, $p=0.0262(t=3, \mathrm{df}=4)$ \\
\hline $9 D$ & $\begin{array}{l}n=3 \text { Sox } 2^{f / f l} \\
n=3 \text { Pdgfra-CreER }{ }^{\mathrm{T} 2}, \text { Sox } 2^{\mathrm{fl} / f \mathrm{l}}\end{array}$ & $\begin{array}{l}\text { Two-tailed unpaired } \\
\text { Student's } t \text { test }\end{array}$ & $\begin{array}{l}\mathrm{CC}^{+} \text {OLs, } p=0.0018(t=7.382, \mathrm{df}=4) \\
\mathrm{CC} 1^{+} \mathrm{BrdU} \mathrm{H}^{+} \text {OLs, } p=0.0175(t=3.904, \mathrm{df}=4) \\
\text { PDGFR } \alpha^{+} \text {OPCS, } p=0.0100(t=4.608, \mathrm{df}=4) \\
\text { PDGFR } \alpha^{+} \mathrm{Ki}^{+} 7^{+} \text {OPCs, } p=0.0007(t=9.604, \mathrm{df}=4)\end{array}$ \\
\hline $9 F$ & $\begin{array}{l}n=3 \text { Sox2 WT + Cuprizone } \\
n=3 \text { Sox2 cK0 + Cuprizone } \\
n=4 \text { Sox2 WT + normal diet }\end{array}$ & $\begin{array}{l}\left.\text { One-way ANOVA }{ }^{* * *} p<0.0001, F_{(2,6)}=100.2\right) \\
\text { with Tukey's post test }\end{array}$ & $\begin{array}{l}\text { Sox2 WT + Cup versus Sox2 cK0 + Cup, }{ }^{* * *} p<0.001 \\
\text { Sox2 WT + Cup versus Sox2 WT + normal diet, }{ }^{* * *} p<0.001 \\
\text { Sox2 cK0 + Cup versus Sox2 WT + normal diet, } p>0.05\end{array}$ \\
\hline $9 H$ & $\begin{array}{l}n=3 \mathrm{~S} 0 \times 2 \mathrm{WT} \\
n=3 \mathrm{~S} 0 \times 2 \mathrm{cK} 0\end{array}$ & $\begin{array}{l}\text { Two-tailed unpaired } \\
\text { Student's } t \text { test }\end{array}$ & $\begin{array}{l}\mathrm{MBP}, p=0.0287(t=3.346, \mathrm{df}=4) \\
\mathrm{SMI} 312, p=0.4334(t=0.9699, \mathrm{df}=4)\end{array}$ \\
\hline $10 C$ & $\begin{array}{l}n=3 \text { Sox2 WT + MOG } \\
n=3 \text { Sox2 cKO + MOG } \\
n=3 \text { Sox2 WT + CFA }\end{array}$ & $\begin{array}{l}\text { One-way ANOVA }\left(* * * p=0.0009, F_{(2,6)}=27.90\right) \\
\text { with Tukey's post test }\end{array}$ & $\begin{array}{l}\text { Sox2 WT + MOG versus Sox2 cKO + MOG, }{ }^{* *} p<0.01 \\
\text { Sox2 WT + MOG versus Sox2 WT + CFA, }{ }^{* * *} p<0.001 \\
\text { Sox2 cK0 + MOG versus Sox2 WT + CFA, } p>0.05\end{array}$ \\
\hline $10 E$ & $\begin{array}{l}n=3 \text { Sox2 WT }+ \text { MOG } \\
n=3 \text { Sox2 CKO + MOG }\end{array}$ & $\begin{array}{l}\text { Two-tailed unpaired } \\
\text { Student's t test }\end{array}$ & $p=0.0019(t=7.303, \mathrm{df}=4)$ \\
\hline $10 F$ & $\begin{array}{l}n=3 \text { Sox2 WT + MOG } \\
n=3 \text { Sox2 CKO + MOG } \\
n=3 \text { Sox2 WT + CFA }\end{array}$ & $\begin{array}{l}\text { One-way ANOVA }\left(^{* * *} p<0.0001, F_{(2,6)}=69.92\right) \\
\quad \text { with Tukey's post test }\end{array}$ & $\begin{array}{l}\text { Sox2 WT + MOG versus Sox2 CKO + MOG, }{ }^{* * *} p<0.001 \\
\text { Sox2 WT + M0G versus Sox2 WT + CFA, }{ }^{* * *} p<0.001 \\
\text { Sox2 cK0 + M0G versus Sox2 WT + CFA, } p>0.05\end{array}$ \\
\hline
\end{tabular}

OPCs and transiently upregulated in newly differentiated, premyelinating OLs along the progression of oligodendroglial lineage during developmental myelination (Fig. 1M) and indicate that Sox 2 may play a crucial role in coordinating multiple steps of oligodendroglial lineage progression.

\section{Sox 2 cKO inhibits developmental myelination and OL} differentiation in the brain of Cnp-Cre, Sox $2^{\mathrm{fl} / \mathrm{fl}}$ mice

The Cre-LoxP-mediated genetic approach was used to study the role of Sox 2 in the progression of oligodendroglial lineage cells. We observed prominent hypomyelination in the forebrain of Cnp-Cre, Sox $2^{\mathrm{fl} / \mathrm{fl}}$ (referred to as Cnp-Sox $2 \mathrm{CKO}$ ) mice, compared with non-Cre Sox ${ }^{\mathrm{fl} / \mathrm{fl}}$ controls on P14 (Fig. $2 A-C$ ). Quantification data showed that the intensity of MBP-immunoreactive signals was significantly reduced in the Cnp-Sox $2 \mathrm{cKO}$ forebrains (Fig. 2D) at the histological level, which was confirmed by Western blot (Fig. 2E). In contrast, the intensity of SMI312 ${ }^{+}$axons was indistinguishable between Cnp-Sox $2 \mathrm{CKO}$ and control mice (Fig. 2D,F), indicating that the observed hypomyelination in the Cnp-Sox $2 \mathrm{cKO}$ brains is less likely due to a diminution of myelinable axons.

Previous studies demonstrate that Cnp-Cre-mediated gene deletion primarily occurs in the later stages of oligodendrocyte development (Dugas et al., 2010; Moyon et al., 2016; Zhao et al., 2016). Using a Cnp-Cre reporter system, we confirmed that $>85 \%$ of PDGFR $\alpha^{+}$OPCs were EYFP-negative in the subcortical white matter tract of $\mathrm{P} 7 \mathrm{Cnp}$-Cre, Rosa26-EYFP reporter mice (Fig. 3A, arrowheads), and almost all $\mathrm{EYFP}^{+}$cells were $\mathrm{O}^{+}$ differentiation-committed late OPCs and/or differentiated OLs (Fig. 3B, arrowheads) (Miron et al., 2011). Consistent with the EYFP expression, Sox 2 was intact in most PDGFR $\alpha{ }^{+}$OPCs (Fig. $3 C$, bottom, arrowheads) but primarily deleted in $\mathrm{CC} 1^{+} \mathrm{OLs}$ (Fig. 3C, bottom, arrows) in the subcortical white matter of CnpSox2 cKO brains.
The hypomyelination phenotype (Fig. 2) and stage-specific Sox 2 deletion (Fig. $3 A-C$ ) suggest that OL differentiation may be affected in the brain of the Cnp-Sox 2 cKO mice. To this end, we used stage-specific markers to analyze OL differentiation: CC1 for OLs, PDGFR $\alpha$ for OPCs, and Olig2 for both OLs and OPCs. The density of Olig $2{ }^{+} \mathrm{CC}{ }^{+}$differentiated OLs was significantly lower in the subcortical white matter of Cnp-Sox $2 \mathrm{cKO}$ mice, whereas the density of Olig2 ${ }^{+} \operatorname{PDGFR} \alpha{ }^{+}$OPCs was statistically similar between the two groups (Fig. $3 D$ ), suggesting a specific perturbation of OL differentiation in the Cnp-Sox $2 \mathrm{cKO}$ brains. We also found more than twofold reduction in the density of TCF712 ${ }^{+}$premyelinating OLs in the subcortical white matter of P14 Cnp-Sox 2 cKO mice (Fig. $3 E, F$ ), indicating a diminished rate of OL generation in the Cnp-Sox $2 \mathrm{cKO}$ brains.

Together, by leveraging the stage-specific Cnp-Cre, Sox $2^{\mathrm{fl} / \mathrm{fl}}$ cKO system, our data demonstrate that Sox 2 positively regulates OL differentiation during developmental myelination in the murine brain.

\section{Conditional Sox 2 ablation does not affect the density of oligodendrocytes in the postnatal spinal cord of P14 Cnp-Cre, Sox $2^{\mathrm{fl} / \mathrm{fl}}$ mice}

Unlike impaired OL differentiation in the brain, the distribution of $\mathrm{CC}^{+}$differentiated OLs (Fig. 4A) and the density of Olig ${ }^{+} \mathrm{CC}^{+}{ }^{+}$differentiated OLs (Fig. $4 B$ ) were comparable in the spinal cord of Cnp-Sox2 cKO mutants from those in the non-Cre Sox $2^{\mathrm{fl} / \mathrm{fl}}$ controls at P14. Western blotting showed that MBP protein expression was similar between the two groups (Fig. $4 C)$. Consistently, qRT-PCR quantification demonstrated that the mRNA levels of the major myelin genes, $M b p, P l p, M a g$, and Mobp, and pan-oligodendroglial lineage cell marker Sox10 was statistically indistinguishable in the spinal cords of Cnp-Sox2 cKO and control mice (Fig. 4D). The unperturbed OL differentiation is unlikely due to the efficiency of Sox 2 deletion because 
P0 corpus callosum near subventricular zone
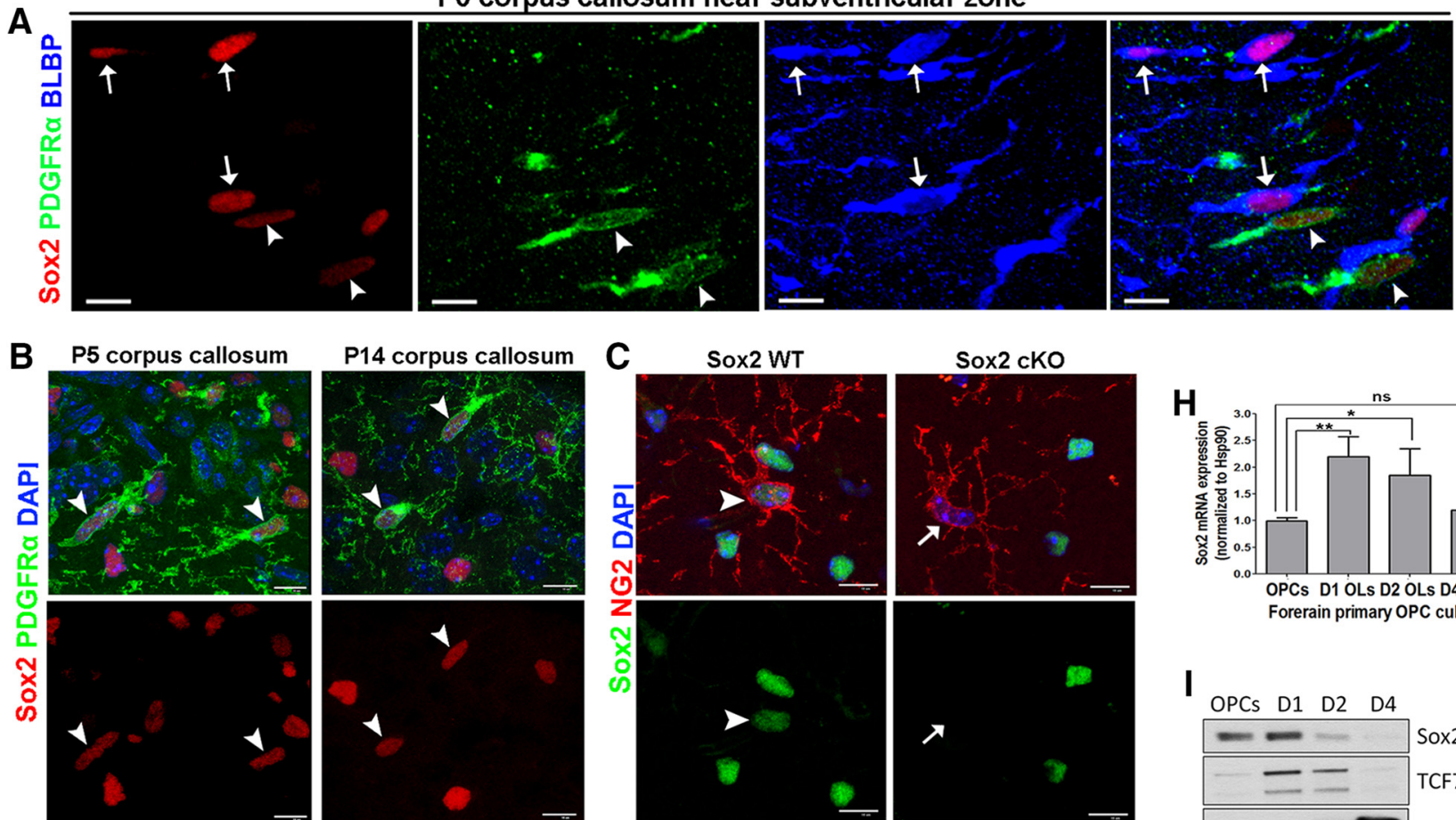

D P14 corpus callosum

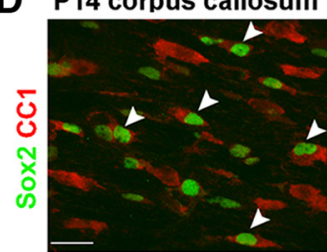

F $\mathrm{P} 10$ corpus callosum $\mathrm{G}$
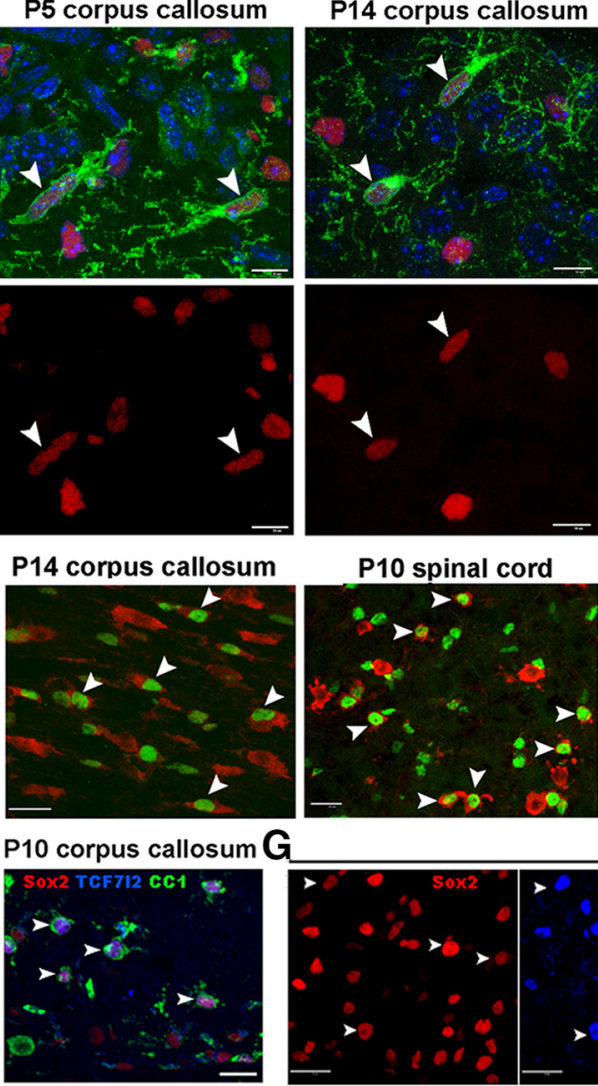

P10 spinal cord
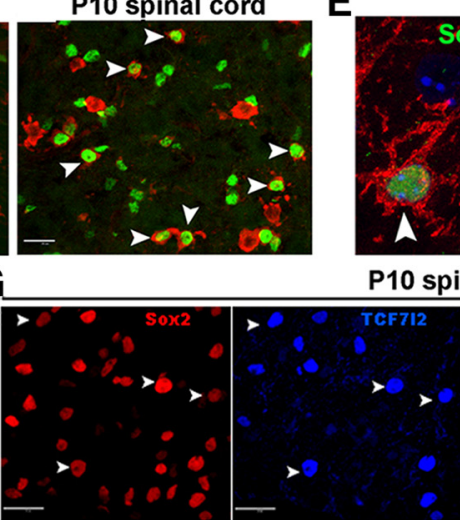

C
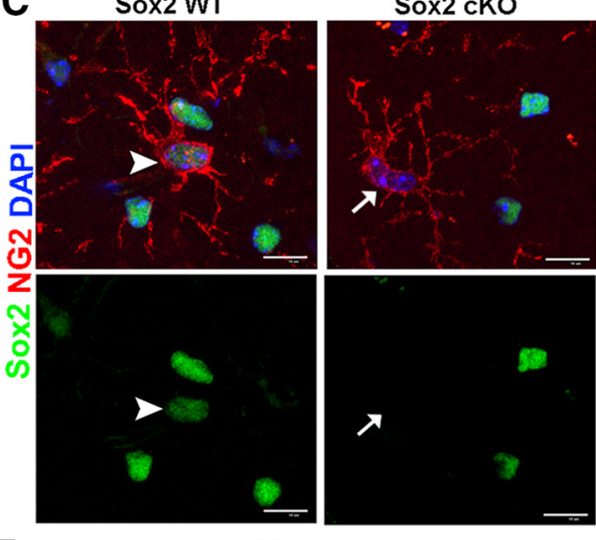

E.

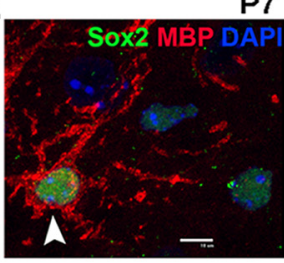

7 brain
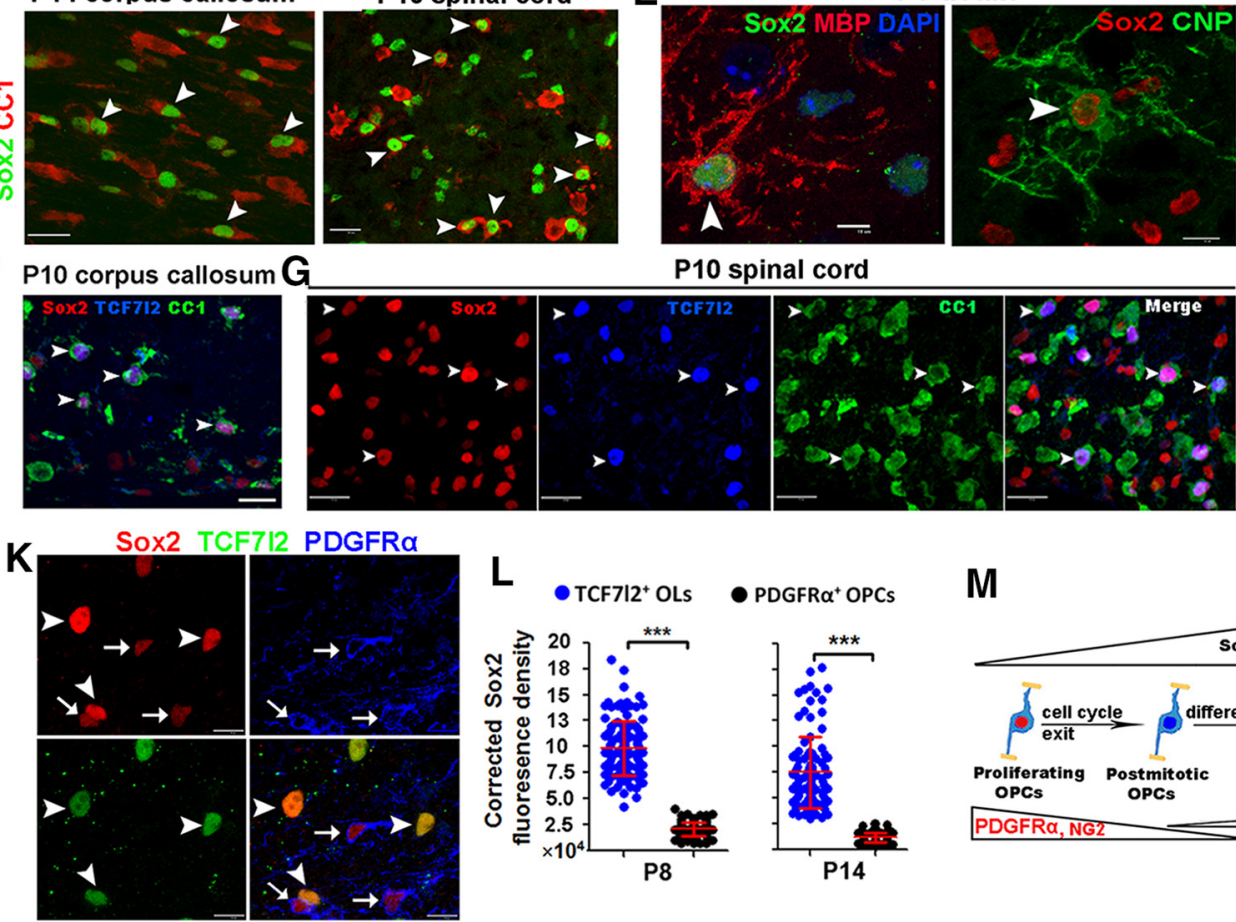

L

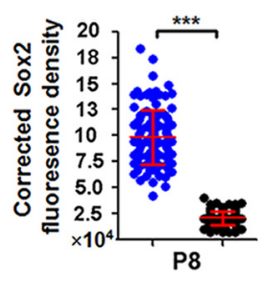

- PDGFRa OPCS

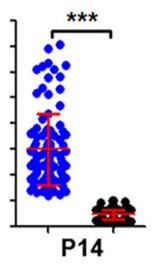

M

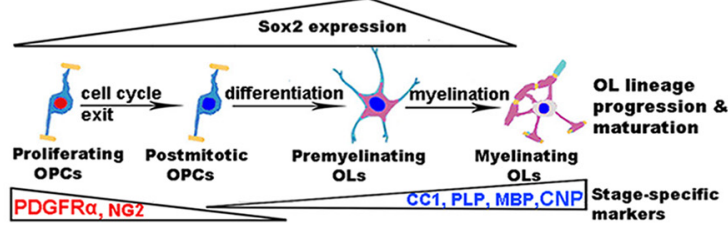

Figure 1. Sox 2 is expressed in postnatal and adult OPCs and transiently upregulated in premyelinating OLs. $A$, Confocal images showing Sox2 expression in PDGFR $\alpha^{+}$OPCs (arrowheads) and brain lipid basic protein (BLBP) ${ }^{+}$astrocytic precursor cells (arrows) in the corpus callosum close to the subventricular zone on postnatal day $\mathrm{PO}$, several days before the presence of differentiated $0 \mathrm{LS}$. $B$, Confocal images showing that Sox2 is expressed in PDGFR $\alpha^{+}$OPCs in the brain corpus callosum at P5 (before OPC differentiation) and P14 (peak OPC differentiation) during developmental myelination. Arrowheads point to Sox2 ${ }^{+}$PDGFR $\alpha^{+}$OPCs. C, Confocal images showing that Sox2 is expressed in adult $\mathrm{NG2}^{+} \mathrm{OPCs}$ at P60 (left panels, arrowheads) and that the expression is abolished in Sox2 cK0 brain of P60 Pdgfr $\alpha$-CreER ${ }^{\mathrm{T} 2}$, Sox2 ${ }^{\mathrm{fl} / \mathrm{fl}}$ mice that had been administered tamoxifen $5 \mathrm{~d}$ before tissue harvest (right panels, arrows). D, Double immunostaining of Sox2 and differentiated $0 \mathrm{~L}$ marker $\mathrm{CC} 1$ in forebrain corpus callosum and spinal cord. Arrowheads point to Sox2 ${ }^{+} \mathrm{CC} 1^{+}$OLs. E, Confocal images showing Sox2 expression in myelin gene CNP-and MBP-positive differentiated OLs (arrowheads). Blue represents DAPI. $\boldsymbol{F}, \boldsymbol{G}$, Triple immunostaining of Sox2, CC1, and TCF7I2 in the corpus callosum (F) and spinal cord (G) showing that Sox2-expressing 0Ls

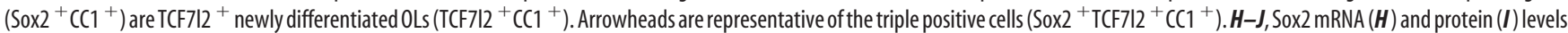
in purified, mouse primary OPCs and differentiating $0 \mathrm{Ls}$ at day 1 (D1), day 2 and day 4 ( $n=3$ at each time point). The differentiation of primary $0 \mathrm{PC}$ is verified by the sharp increase of MBP protein $(I)$ and mRNA $(J)$ from D1-D4. Sox2 protein expression is overlapped with TCF712 in differentiating 0Ls $(I) . K, L$, Representative images of triple immunostaining of Sox2, TCF712, and PDGFR $\alpha$ in the forebrain corpus callosum $(\boldsymbol{K})$ and quantification of corrected Sox2 fluorescence density in TCF712 ${ }^{+}$newly differentiated OLs and PDGFR $\alpha^{+}$OPCs in P8 and P14 brains ( $(\boldsymbol{L})$. $\boldsymbol{K}$, Arrowheads and arrows point to Sox2 ${ }^{+} \mathrm{TCF}_{\mathrm{NI} 2}{ }^{+}$OLs and Sox2 ${ }^{+}$PDGFR $\alpha^{+}$OPCS, respectively. $M$, Schematic drawing illustrating Sox2 expression patterns along the progression and maturation of oligodendroglial lineage. OPC markers PDGFR $\alpha$ and NG2 are progressively downregulated, whereas OL markers CC1, proteolipid protein (PIp), MBP, and CNPase (CNP) are progressively (Figure legend continues.) 

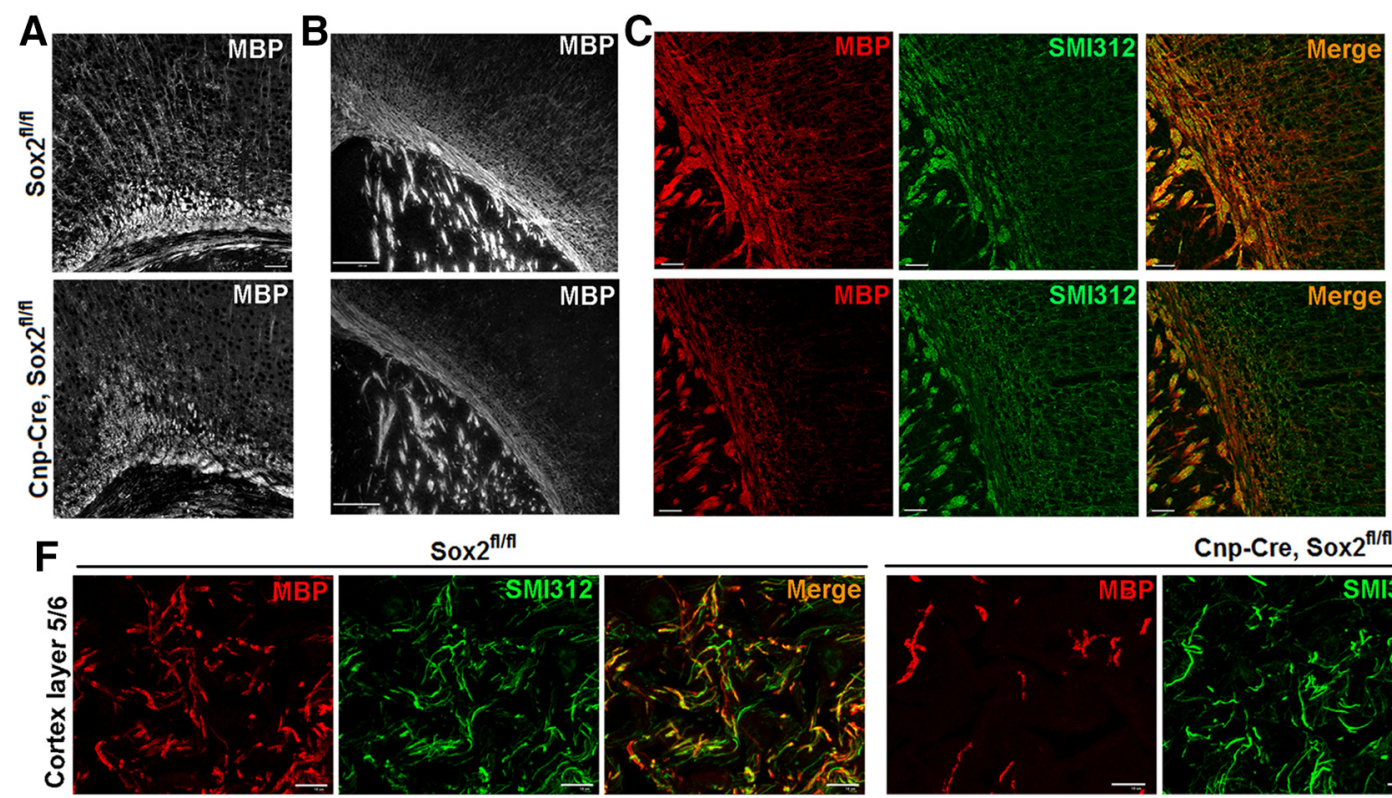

$\operatorname{Sox} 2^{\text {fl/fi }}$
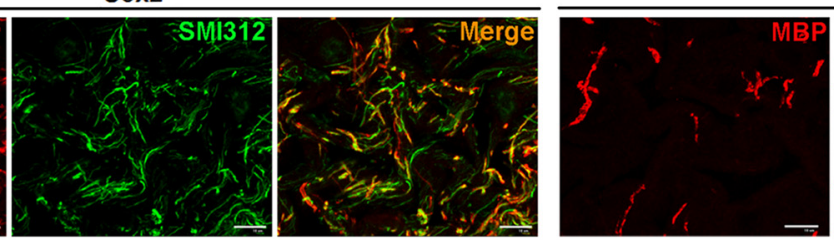

Cnp-Cre, Sox $2^{\text {fl/f }}$
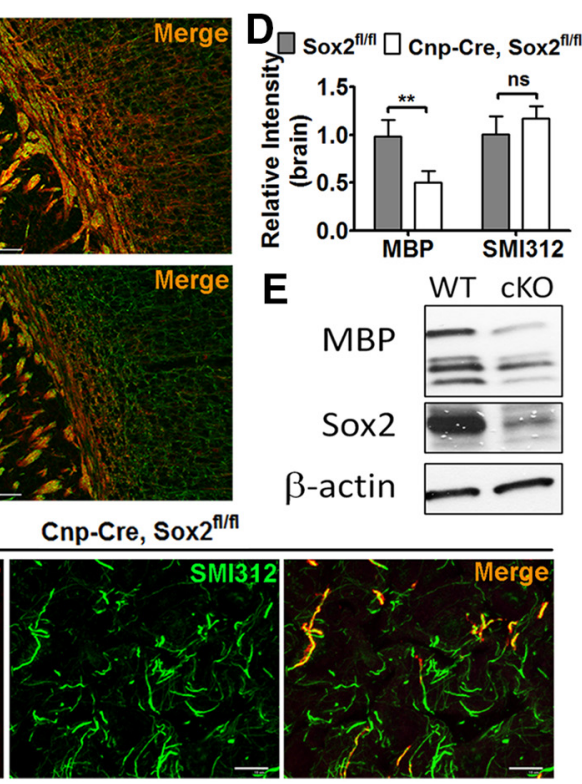

Figure 2. Cnp-Cre, Sox ${ }^{\mathrm{f} / \mathrm{fl}}$ mice display brain hypomyelination on postnatal day $14 . \boldsymbol{A}, \boldsymbol{B}$, Grayscale images of MBP immunostaining showing decreased density of myelin fibers in the corpus callosum at the cingular cortical areas $(\boldsymbol{A})$ and in the subcortical white matter tracts $(\boldsymbol{B})$. $\boldsymbol{C}$, Single and merged channels of confocal images of MBP and SMI312 in the subcortical white matter and the overlaying cortex. Most SMI312 ${ }^{+}$axons (green) lack MBP ${ }^{+}$myelin (red) in the Cnp-Cre, Sox $2^{\mathrm{fl} / f \mathrm{l}}$ mice (lower right), in sharp contrast to extensive colabeling of MBP and SMI312 in the Sox2 $2^{\mathrm{fl} / f \mathrm{l}}$ littermate controls (top right). D, Quantification of relative densities of MBP and SMI312 immunoreactive signals $\left(n=4\right.$ Cnp-Cre, Sox $2^{\mathrm{ff} / \mathrm{fl}}, n=5$ Sox ${ }^{\mathrm{f} / / \mathrm{fl}}$ mice). ${ }^{* *} p<0.01$ (two-tailed Student's $t$ test). $\boldsymbol{E}$, Representative image of Western blotting analyses of Sox2 and MBP protein from Cnp-Cre, Sox $2^{\mathrm{flffl}}$ (cKO) and Sox $2^{\mathrm{f} / / \mathrm{fl}}$ (WT) forebrains. $\beta$-actin serves as an internal loading control. $\boldsymbol{F}$, High-power confocal images of MBP and SMI312 in the cortical areas of layers 5/6 of P14 Cnp-Cre, Sox ${ }^{\text {fl/fl }}$ and Sox ${ }^{\mathrm{fl} / \mathrm{fl}}$ control brains. Scale bars: $\boldsymbol{B}, 200 \mu \mathrm{m} ; \boldsymbol{C}, 50 \mu \mathrm{m}, \boldsymbol{F}, 10 \mu \mathrm{m}$.

the levels of Sox 2 protein (Fig. 4C) and mRNA (Fig. 4D) were significantly decreased in the spinal cord, and Sox2 expression was abolished in all $\mathrm{CCl}^{+}$oligodendrocytes at the histological level (Fig. 4E). Our data collected from the P14 spinal cord of Cnp-Sox 2 cKO mutants are in agreement with a recent study showing that Sox 2 disruption elicited by the ubiquitously expressed CAG-CreER ${ }^{\mathrm{T} 2}$ does not affect the number of differentiated OLs in the postnatal spinal cord at P14 (Zhao et al., 2015).

Sox 2 ablation specifically in OPCs reveals an essential role of Sox 2 in OPC population expansion

The observation that Sox 2 was expressed in OPCs from postnatal to adult CNS (Fig. 1) led us to hypothesize that Sox2 is additionally required for OPC population expansion. Because the CnpSox 2 cKO paradigm did not induce efficient Sox 2 deletion in OPCs in the subcortical white matter, we used Pdgfr $\alpha-C r e E R^{T 2}$, Sox ${ }^{\mathrm{fl} / \mathrm{fl}} \mathrm{cKO}$ to ablate Sox 2 specifically in OPCs.

Tamoxifen was injected intraperitoneally to the $P d g f r \alpha$ $C r e E R^{T 2}$, Sox $2^{\mathrm{fl} / \mathrm{fl}}$ mice (referred to as Pdgfr $\alpha$-Sox $2 \mathrm{cKO}$ ) and non-Cre Sox $2^{\mathrm{fl} / \mathrm{fl}}$ control mice on P6 and P7. We found that $88.9 \%( \pm 9.4 \%$, SD) OPCs in the subcortical white matter had no detectable Sox 2 expression on P9, $2 \mathrm{~d}$ after the last tamoxifen treatment (Fig. $5 A$, right panels, arrowheads), in sharp contrast to the low level expression of Sox 2 in all OPCs in control mice (Fig. $5 A$, left panels, arrowheads). On P14, 1 week after the last tamoxifen injection, the density of Sox $10^{+}$PDGFR $\alpha^{+}$OPCs (Fig. 5B) in the subcortical white matter was significantly lower in the Pdgfr $\alpha$-Sox 2 cKO mutants compared with non-Cre Sox $2{ }^{\mathrm{fl} / \mathrm{fl}}$ controls (Fig. $5 C$, top). Consistent with the histological quantification,

(Figure legend continued.) upregulated. One-way ANOVA with Tukey's post hoc test $(\boldsymbol{H})$ and two-tailed Student's $t$ test $(\boldsymbol{L}):{ }^{*} p<0.05 ;{ }^{* *} p<0.01 ;{ }^{* * *} p<0.001$ (for the details of the statistical analyses for all figures, see Table 1). Scale bars: $\boldsymbol{A}-\boldsymbol{C}, \boldsymbol{E}, \boldsymbol{K}, 10 \mu \mathrm{m} ; \boldsymbol{D}, \boldsymbol{F}, \mathbf{G}, 20 \mu \mathrm{m}$.
qRT-PCR results showed that the mRNA level of OPC marker PDGFR $\alpha$ was also significantly diminished (Fig. 5C, bottom).

OPC population is mainly expanded during the first postnatal week in the murine brain. To determine the role of Sox2 in OPC expansion during this early postnatal stage, we deleted Sox 2 in OPCs on P1, P2, and P3 by tamoxifen injections and analyzed OPC population on P8 (Fig. 5D). Our data showed that the density of Sox $10^{+}$PDGFR $\alpha^{+}$OPCs in the subcortical white matter (Fig. 5E) was significantly diminished in the Pdgfr $\alpha$-Sox $2 \mathrm{cKO}$ mice compared with that in the non-Cre controls (Fig. $5 F$ ).

We speculate that diminished OPC population size observed in the Pdgfr $\alpha$-Sox $2 \mathrm{cKO}$ brains will result in decreased OL differentiation and myelination. RNA sequencing was used to identify differentially expressed genes at the transcriptional level between Pdgfr $\alpha$-Sox 2 cKO and control forebrains on P14 (tamoxifen injections on P6 and P7). Gene ontology analysis of the differentially expressed genes (Table 2) showed significant enrichments in the biological processes of OL development, differentiation, and myelination (Fig. 5G). qRT-PCR quantification confirmed that the mRNA levels of myelination- and OL differentiationrelated genes were significantly reduced in the Pdgfr $\alpha$-Sox 2 cKO forebrains (Fig. $5 \mathrm{H}$ ). The density of Sox $10^{+} \mathrm{CC} 1^{+}$differentiated OLs was also significantly attenuated at the histological level (Fig. $5 I$ ). Together, our data suggest that Sox2 is required for OPC population expansion in the postnatal murine brain.

\section{Sox 2 controls OPC population supply by regulating OPC cell} proliferation but not survival

OPC homeostasis is regulated by OPC proliferation, survival, or both. We analyzed proliferation rate of brain OPCs of P14 Pdgfr $\alpha$-Sox 2 cKO and control mice, both of which had received tamoxifen on P6 and P7. Two hours of EdU pulse labeling demonstrated that the number of Sox $10^{+} \mathrm{EdU}^{+}$proliferating OPCs (Fig. 6A) was significantly less in the Pdgfr $\alpha$-Sox 2 cKO brain than 

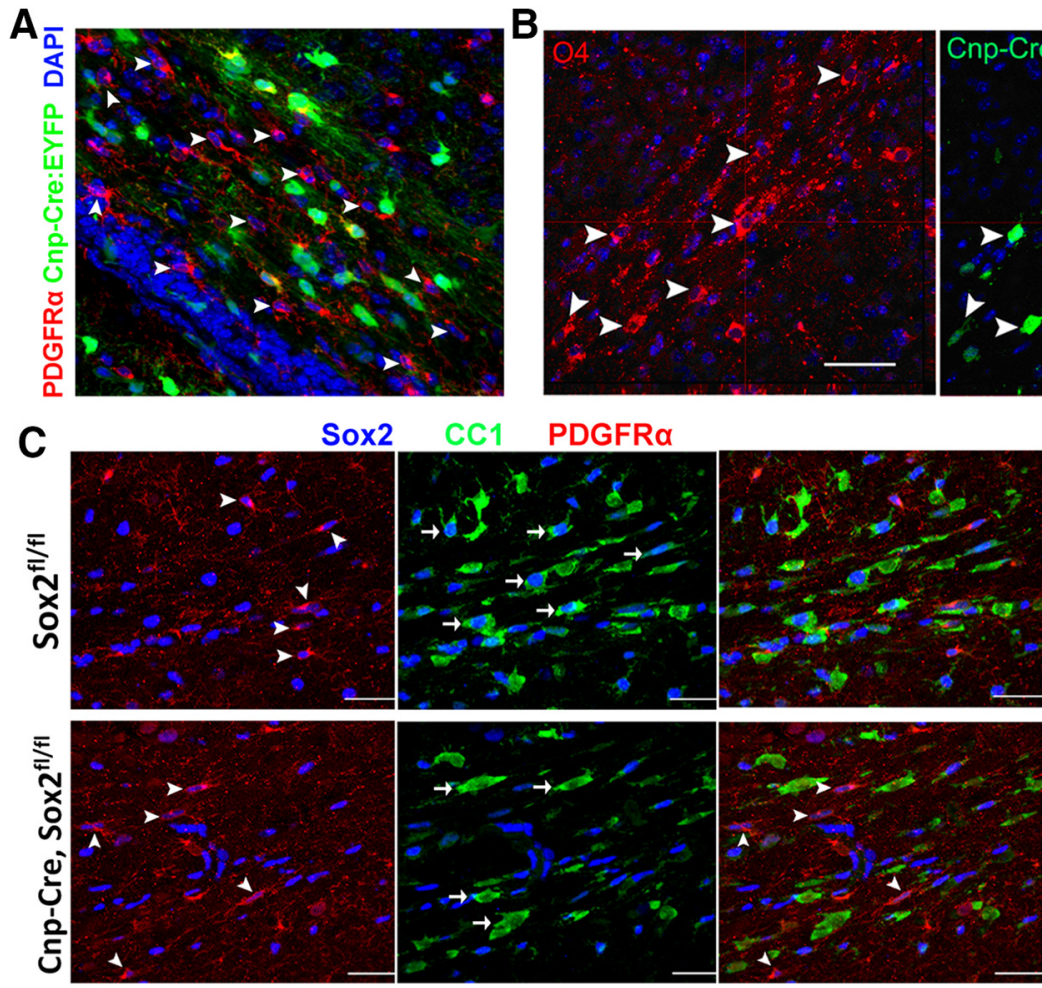
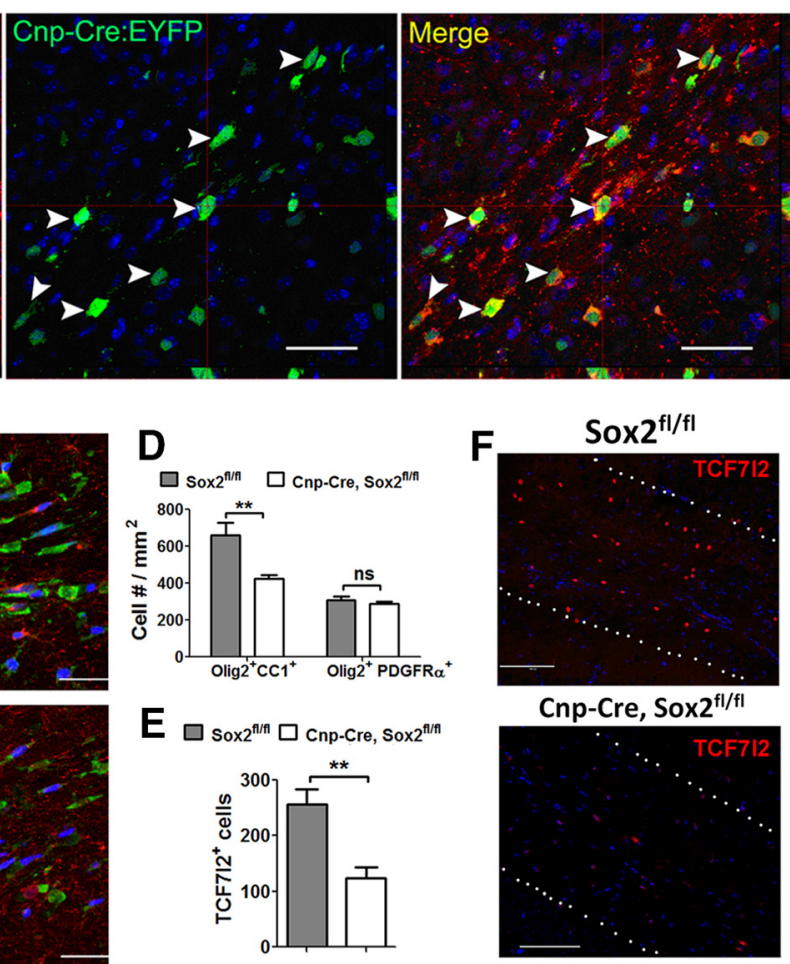

Figure 3. Oligodendrocyte differentiation is inhibited in the brain of $C n p$-Cre Sox ${ }^{\mathrm{fl} / \mathrm{fl}}$ mice. $\boldsymbol{A}$, Fate-mapping of $\mathrm{Cnp}$-Cre-expressing cells in the subcortical white matter of P7 Cnp-Cre, Rosa-EYFP mice. Many PDGFR $\alpha^{+}$OPCs are EYFP ${ }^{-}$(arrowheads). $\boldsymbol{B}$, Many EYFP ${ }^{+}$fate-mapped cells are $04^{+}$differentiation-committed, late OPCs, and/or immature oligodendrocytes (arrowheads) in the subcortical white matter of P7 Cnp-Cre, Rosa-EYFP mice. C, Triple immunostaining of CC1, PDGFR $\alpha$, and Sox2 in the corpus callosum. Arrows and arrowheads point to representative CC ${ }^{+} 0 \mathrm{Ls}$ and PDGFR $\alpha{ }^{+}$OPCS, respectively. In the Sox2 CK0 corpus callosum (bottom), many OPCs retain Sox2 expression, whereas no OLs have detectable Sox2. $\boldsymbol{D}$, Quantification of Olig2 ${ }^{+} \mathrm{CC} 1^{+}$OLs and 0lig2 ${ }^{+}$PDGFR $\alpha{ }^{+}$OPCs in the subcortical white matter tract. Olig2 is a pan-oligodendroglial marker expressed in both OPCs and $0 \mathrm{Ls}$. $\boldsymbol{E}, \boldsymbol{F}, \mathrm{Density}\left(\mathrm{Cell}\right.$ no./mm $\left.{ }^{2}\right)(\boldsymbol{E})$ and representative images $(\boldsymbol{F})$ of TCF7I2-expressing, newly differentiated $0 \mathrm{Ls}$ in the subcortical white matter tracts $\left(\boldsymbol{F}\right.$, dotted areas). $\boldsymbol{C}-\boldsymbol{F}$, Animals are P14 old. $\boldsymbol{D}, \boldsymbol{E}, n=4$ Cnp-Cre Sox $2^{\text {fl/fl }}$ and $n=6$ Sox ${ }^{\text {fl/fl }}$. ${ }^{* *} p<0.01$ (two-tailed Student's $t$ test). Scale bars: $\boldsymbol{A}-\boldsymbol{C}, 50 \mu \mathrm{m} ; \boldsymbol{F}, 100 \mu \mathrm{m}$.

that in controls (Fig. 6B, left). The decreased proliferative rate was confirmed by another proliferation-related antigen Ki67 (Fig. 6B, right). The survival of OPCs, however, was not affected as demonstrated by no significant differences in the number of active Caspase $3^{+}$Sox $10^{+}$(Fig. 6C,D, left) and TUNEL ${ }^{+}$Sox $10^{+}$(Fig. $6 D$, right) apoptotic oligodendroglial lineage cells between the two groups. We also did not observe differences in the density of Caspase $3^{+}$Sox $10^{+}$apoptotic oligodendroglial lineage cells in the subcortical white matter near the subventricular zone in the forebrain of P8 Pdgfr $\alpha$-Sox 2 cKO that had been administered tamoxifen on P1, P2, and P3 (4.6 $\pm 0.8 / \mathrm{mm}^{2}$ in Pdgfr $\alpha-S o x 2 \mathrm{cKO}$ vs $5.4 \pm 1.2 / \mathrm{mm}^{2}$ in Sox ${ }^{\mathrm{fl} / \mathrm{fl}}, n=3$ each group).

Previous study suggests that Sox 2 critically regulates neural stem cell survival in the subgranular zone (SGZ) (Favaro et al., 2009) and SVZ (Feng et al., 2013) in the murine brain. We ablated Sox 2 in all Sox2-positive cells (including Sox $2{ }^{+}$neural stem cells and oligodendroglial lineage cells) by using Sox2-CreER ${ }^{T 2}$, So $\times 2^{\mathrm{fl} /+}$ cKO system (the Sox2-CreER ${ }^{\mathrm{T} 2}$ is a homologous knock-in transgene). Tamoxifen was administered on P6 and P7, and cell survival was analyzed on P14. Our quantification data showed that the number of active Caspase $3^{+}$apoptotic cell in the SGZ and SVZ was significantly increased in Sox2-CreER ${ }^{\mathrm{T} 2}$, Sox ${ }^{\mathrm{fl} /+}$ mice (referred to as Sox2-Sox2 cKO) $\left(156.6 / \mathrm{mm}^{2}\right.$ in Sox2-Sox2 cKO SGZ vs 11.4/ $\mathrm{mm}^{2}$ in control SGZ, $n=3, p<0.0001 ; 24.7 / \mathrm{mm}^{2}$ in Sox2-Sox2 SVZ vs $4.2 / \mathrm{mm}^{2}$ in control SVZ, $n=3, p=0.0021$, two-tailed Student's $t$ test). The altered cell survival detected in the SGZ and SVZ stem cell niche of the Sox2-Sox 2 cKO mice is consistent with previous publications (Favaro et al., 2009; Feng et al., 2013), which also supports the effectiveness of our cell survival analysis. Double immunohistochemistry of active Caspase 3 and lineage-specific markers demonstrated that apoptotic cells in the SGZ (Fig. 6G) and SVZ (data not shown) were $\mathrm{GFAP}^{+}$neural stem cells but not doublecortin ${ }^{+}$neuroblasts nor $\mathrm{HuC} / \mathrm{D}^{+}$neurons.

In agreement with the cell survival analysis in the Pdgfr $\alpha$-Sox2 cKO system (Fig. 6C,D), we did not notice any changes in the number of active Caspase $3^{+}$Sox $10^{+}$apoptotic oligodendroglial lineage cells in SGZ and SVZ between Sox2-Sox2 cKO and nonCre controls (Fig. 6E,F, right panels). These data suggest that Sox2's role in cell survival is cell type-dependent: it regulates neural stem cell survival, but it is dispensable for oligodendroglial lineage cell survival.

\section{Oligodendrocyte differentiation and myelination are}

impaired in the Sox10-Cre, Sox $2^{\mathrm{fl} / \mathrm{fl}}$ mutants, even in the later stages of brain development

Using stage-specific Sox 2 cKO paradigms, our experimental data suggest that Sox 2 regulates brain myelination by coordinating upstream OPC proliferation and downstream OL differentiation. Therefore, we predict that Sox $2 \mathrm{cKO}$ in all oligodendroglial lineage cells (both OPCs and OLs) will lead to the inhibition of OPC expansion, OL differentiation, and brain myelination, even in the later stages of brain development. To support this prediction and also to strengthen our conclusion drawn from Cnp-Sox $2 \mathrm{cKO}$ and Pdgfr $\alpha$ Sox 2 cKO mutants, we used Sox10-Cre (Matsuoka et al., 2005) to ablate Sox 2 in all oligodendroglial lineage cells and analyzed brain myelination and OL differentiation at later developmental ages. 

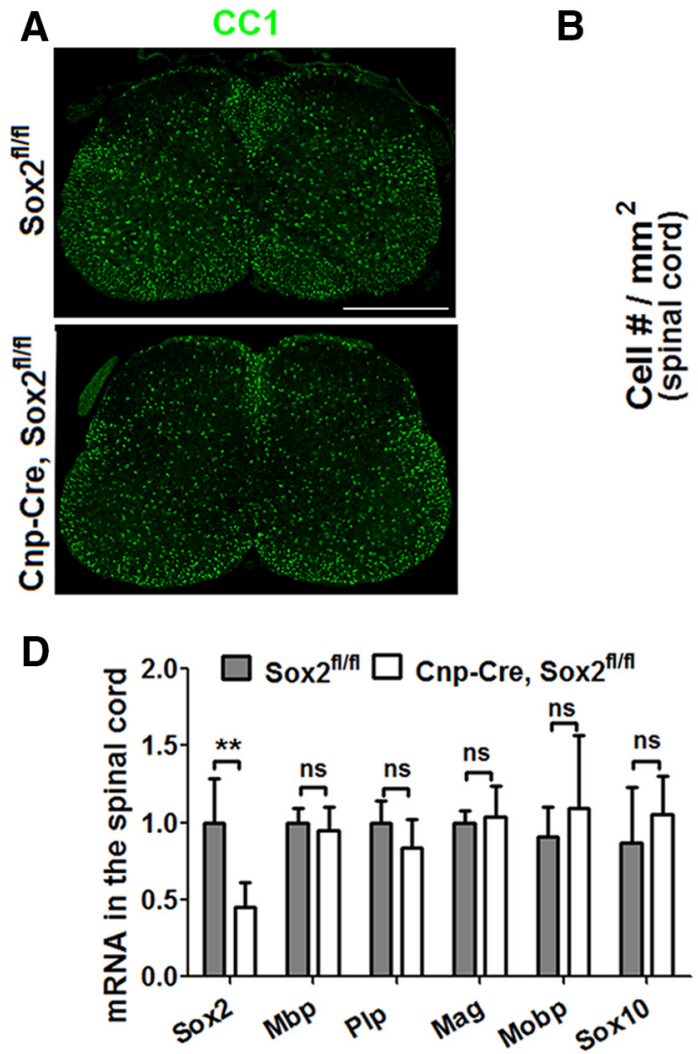

B

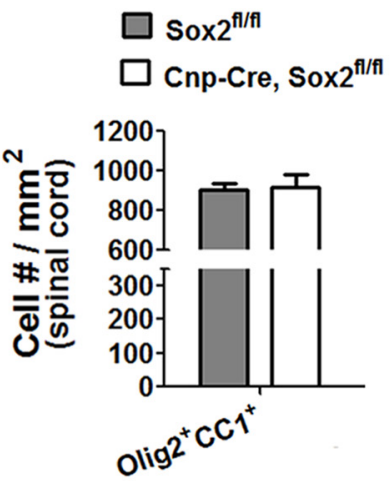

C

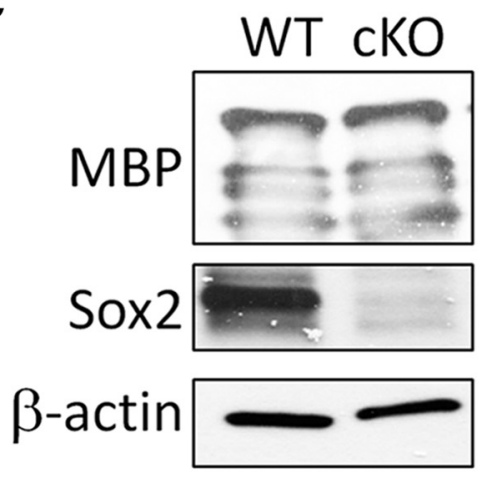

E

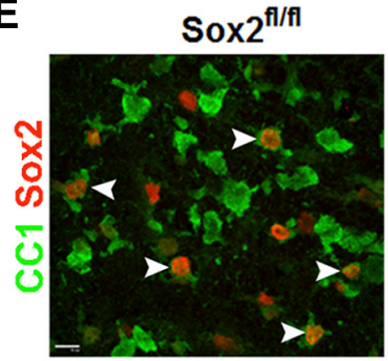

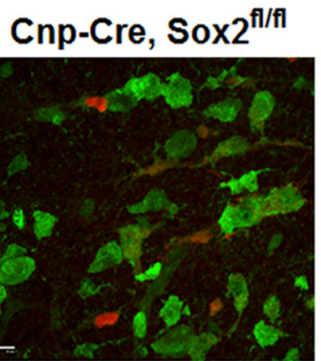

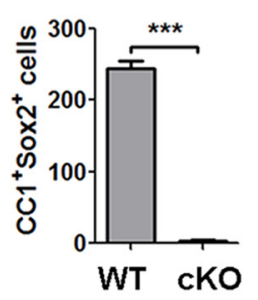

Figure 4. Oligodendrocyte number appears normal in the spinal cord of P14 Cnp-Cre Sox ${ }^{\text {fl/fl }}$ mice. $\boldsymbol{A}$, Low-power confocal images showing the distribution of CC ${ }^{+}$differentiated $0 \mathrm{Ls}$ in the

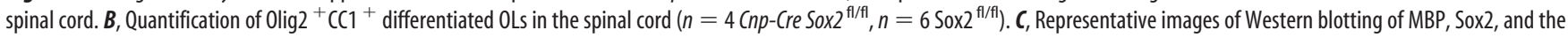
internal loading control $\beta$-actin in the spinal cord. D, qRT-PCR quantification of mRNA levels of Sox2, Mbp, proteolipid protein (PIp), myelin-associated protein (Mag), myelin-associated oligoden-

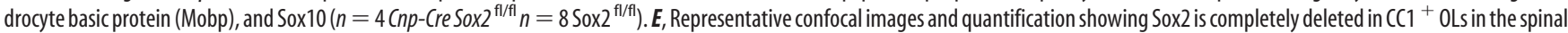

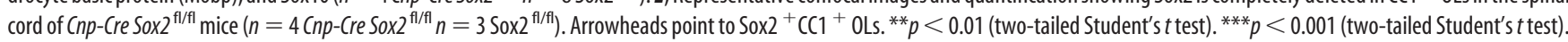
Spinal cord tissues are harvested at P14 old. Scale bars: $A, 500 \mu \mathrm{m} ; \boldsymbol{E}, 10 \mu \mathrm{m}$.

The Sox10-Cre, Sox $2^{\text {fl/fl }}$ (referred to as Sox10-Sox2 cKO) mice developed severe tremors and ataxia, typical phenotypes reminiscent of CNS hypomyelination, by the third postnatal weeks P21 (Movie 1), whereas littermate control mice did not show any of the aforementioned behavioral phenotypes (Movie 2). Behavioral testing demonstrated that Sox $10-$ Sox 2 cKO mice displayed severe motor function impairment, evidenced by significantly less retention time on the accelerating rod (129.2 s, non-Cre Sox $2^{\mathrm{f} / \mathrm{fl}}$ control mice vs $2.1 \mathrm{~s}$, Sox10-Sox 2 cKO mice) (Fig. 7A).

The efficiency of Sox 2 ablation in the Sox10-Sox 2 cKO CNS was nearly $100 \%$ in the oligodendroglial lineage cells, including OPCs (Fig. 7B) and OLs (data not shown) assessed on P21. The density of $\mathrm{MBP}^{+}$myelin fibers in the Sox 10-Sox 2 cKO brains was reduced by $50 \%$ of that in littermate controls, whereas the density of SMI $312^{+}$axons was similar between the two groups (Fig. 7C). Western blotting (Fig. 7D, left) demonstrated that the protein levels of MBP, CNP, and MAG were all significantly decreased in the Sox 10-Sox 2 cKO brains compared with controls (Fig. $7 D$, right). In line with the Western blotting data, qRT-PCR quantification showed that the transcription levels of $M b p$ and mature OL-specific Plp isoform (exon3b containing Plp, $P l p-E 3 b)$ were reduced by $>50 \%$ of those in the control brain on P21 (Fig. 7E). Immunohistochemical analysis demonstrated that the numbers of $\mathrm{CCl}^{+}$mature OLs (Fig. 7F) and PDGFR $\alpha^{+}$OPCs were significantly decreased in the corpus callosum of P21 Sox10-Sox2 cKO mice (Fig. 7G). Haploinsufficiency of Sox 2 in regulating brain oligodendroglial development was not observed, evidenced by the unaltered numbers of OPCs and OLs in brains between Sox $2^{\mathrm{fl} / \mathrm{fl}}$ and Sox2 one-allele cKO (Sox10-Cre, Sox $2^{\mathrm{f} / /+}$ ) mice (Fig. $7 G$ ).

Consistent with previous data derived from the embryonic and early postnatal spinal cord (Hoffmann et al., 2014), the distribution of Olig ${ }^{+}$cells was similar between Sox10-Sox 2 cKO and Sox $2^{\mathrm{fl} / \mathrm{fl}}$ littermate controls within the spinal cord cross sections (Fig. $7 \mathrm{H}$ ), but the density of Olig $1^{+}$cells decreased by $30 \%$ $\left(625 \pm 66 / \mathrm{mm}^{2}\right.$ in Sox10-Sox $2 \mathrm{cKO}, 430 \pm 47 / \mathrm{mm}^{2}$ in controls, two-tailed Student's $t$ test, $p=0.0029, t=4.82, \mathrm{df}=6$ ) at the weaning age of P21, suggesting a dispensable role of Sox 2 in oligodendrocyte migration (Hoffmann et al., 2014) and an essential role in oligodendrocyte production. Our quantification data showed that the densities of PDGFR $\alpha^{+}$OPCs and $\mathrm{CC}^{+}$OLs were significantly reduced in the Sox10-Sox 2 cKO spinal cord than those in Sox $2^{\mathrm{f} / \mathrm{fl}}$ controls on P21 (Fig. 7I). Toluidine blue staining of semithin sections (Fig. $7 J$ ) and transmission electron microscopic images of ultrathin sections (Fig. $7 \mathrm{~K}$ ) showed that the myelinated axons were substantially fewer in the corticospinal tract of Sox10-Sox2 cKO mutants compared with littermate controls on P28, which is in line with the decreased density of mature OLs in the Sox10-Sox 2 cKO spinal cord.

Collectively, the in vivo data derived from the Cnp-Sox $2 \mathrm{cKO}$, Pdgfr $\alpha$-Sox 2 cKO, and Sox10-Sox 2 cKO paradigms unequivocally demonstrate that Sox2 plays an essential role in regulating oligodendroglial lineage progression and maturation during brain developmental myelination. 
Tamoxifen at $\mathrm{P} 6 / \mathrm{P7}$, analysis at $\mathrm{P9}$
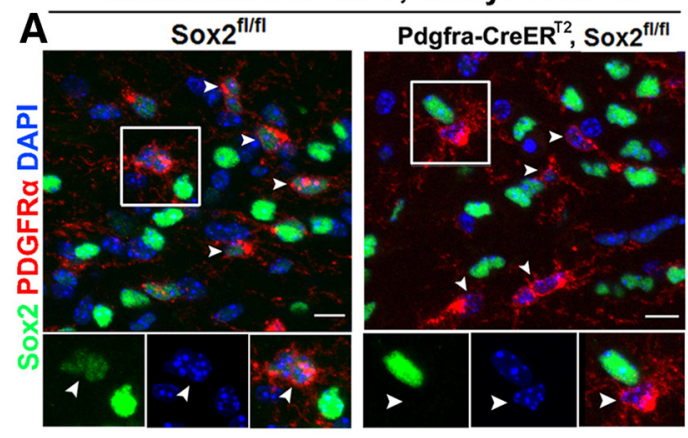

Tamoxifen at $\mathrm{P} 6 / \mathrm{P} 7$, analysis at $\mathrm{P} 14$
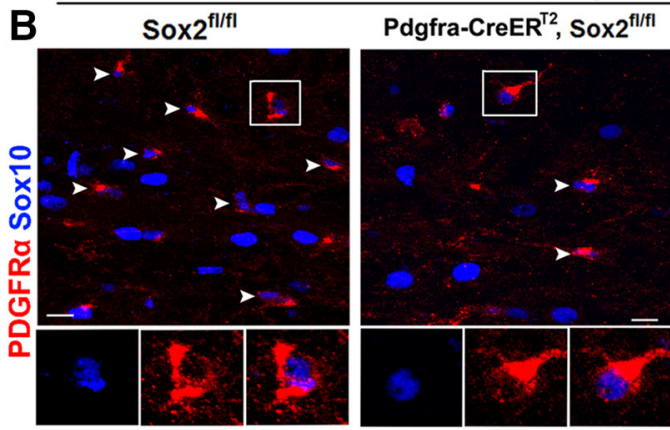

Tamoxifen at P1-P3, analysis at P8
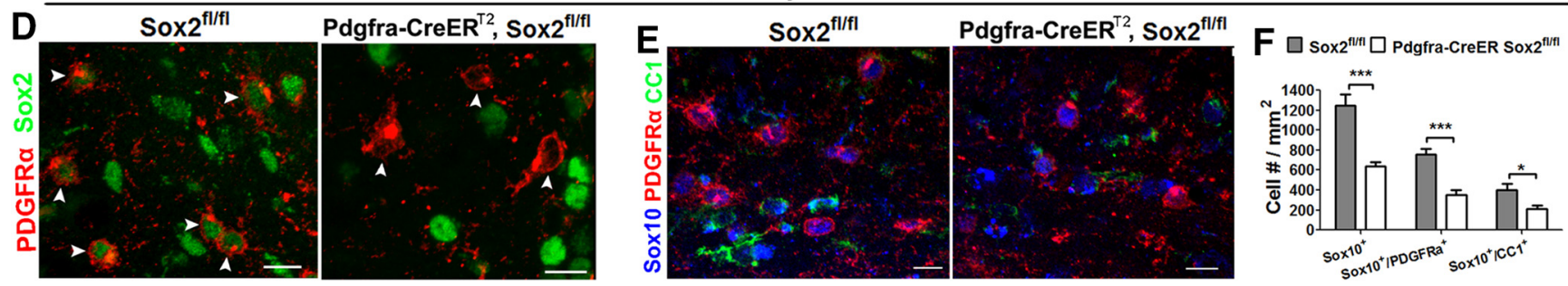

G

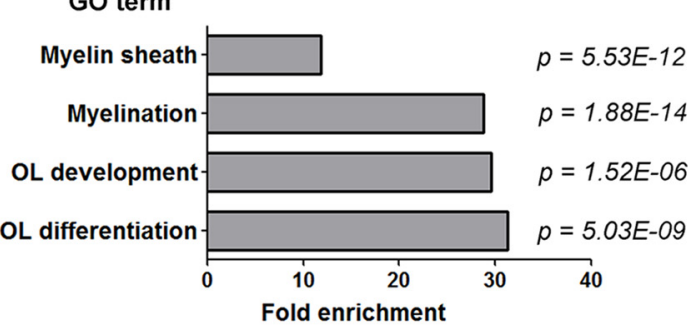

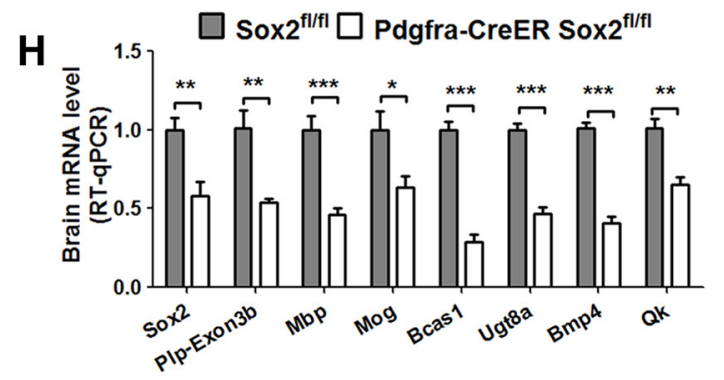
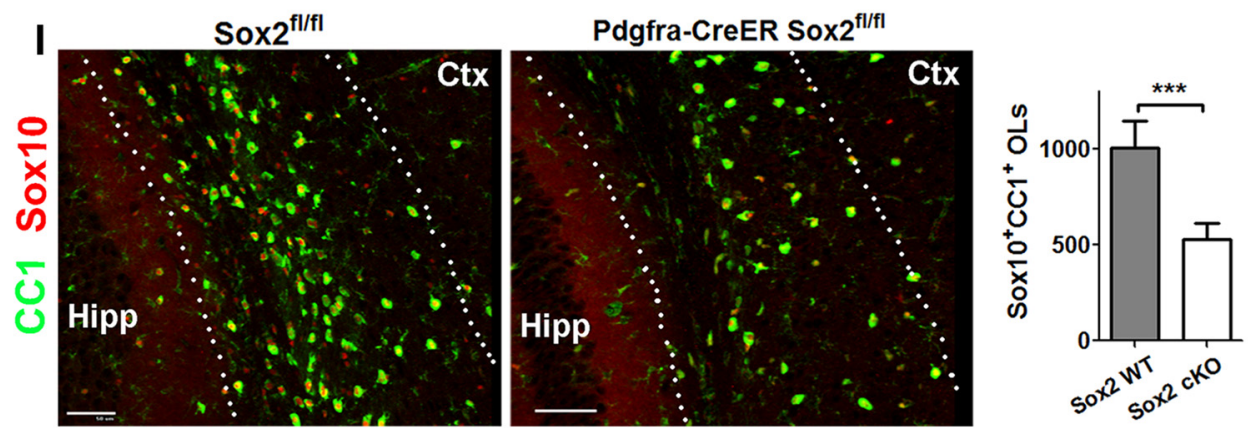

Figure 5. Sox 2 is required for OPC population expansion. $\boldsymbol{A}$, Confocal images depicting Sox2 expression in PDGFR $\alpha^{+}$OPCs (left panels, arrowheads) in the corpus callosum of P9 Sox ${ }^{\mathrm{fl} / \mathrm{fl}}$ mice (left panels, arrowheads), and Sox2 is abolished in PDGFR $\alpha{ }^{+}$OPCs in the corpus callosum of Pdgfr $\alpha$-Cre:Sox2 cK0 mice (right panels, arrowheads). Bottom, Boxed areas represent higher magnification. $\boldsymbol{B}$, Representative confocal images of Sox $10^{+} \mathrm{PDGFR} \alpha{ }^{+}$OPCs (arrowheads) in the P14 corpus callosum. Bottom, Boxed areas represent higher magnification. $\boldsymbol{C}$, Histological quantification of Sox $10^{+}$PDGFR $\alpha{ }^{+}$OPCS and Sox $10^{+}$pan-oligodendroglial lineage cells in the subcortical white matter (top, $n=7$, Pdgfr $\alpha$-Sox2 cK0, $n=7$ control) and qRT-PCR quantification of Sox2 and Pdgfr $\alpha$ mRNA levels in the P14 forebrains (bottom, $n=4$, Pdgfr $\alpha$-Cre:Sox2 cK0; $n=4$, control). D, Immunostaining showing that Sox2 is ablated in PDGFR $\alpha{ }^{+}$OPCs in the corpus callosum of P8 Pdgfr $\alpha$-Cre:Sox2 CK0 mice that had received tamoxifen at P1-P3 (right, arrowhead). $\boldsymbol{E}, \boldsymbol{F}$, Representative images of triple immunostaining of Sox10, PDGFR $\alpha$, and CC1 (E) and quantification of marker ${ }^{+}$cells $(\boldsymbol{F})$ in the P8 corpus callosum. $N=3$ Sox $2^{\mathrm{fl} / \mathrm{fl}}, n=3$ Pdgfr $\alpha$-Sox2 cK0. G, Significant enriched gene ontology terms from gene ontology analyses using the differentially expressed genes derived from RNA-sequencing (Table 2). RNA was extracted from forebrains of P14 mice that had been treated with tamoxifen at P6 and P7. $\boldsymbol{H}$, qRT-PCR quantification of P14 forebrain mRNA showing significant decrease of Sox2, and myelination-related genes, Plp-Exon3b (only expressed in myelinating OLs), Mog, Bcas1, Ugt8a, Bmp4, and Qk, which is identified as the antigen recognized by clone CC1 (Bin et al., 2016). $N=6$ each group. I, Immunostaining and quantification of CC1 and Sox10 in the subcortical white matter tracts (dotted areas) of P14 forebrain (tamoxifen on P6 ad P7). Hipp, Hippocampus; Ctx, cortex ( $n=7$ each group). ${ }^{*} p<0.05$ (two-tailed Student's $t$ test). ${ }^{* *} p<0.01$ (two-tailed Student's $t$ test). ${ }^{* * *} p<0.001$ (tw0-tailed Student's $t$ test). Scale bars: $A, B, D, E, 10 \mu \mathrm{m} ; I, 50 \mu \mathrm{m}$.

Sox 2 expression in oligodendroglial lineage cells during remyelination after chemical-induced and autoimmunity-induced demyelination

We assessed Sox 2 expression in both cuprizone-induced demyelination and MOG-peptide $35-55$-induced EAE animal models
(Guo et al., 2011). In the cuprizone-induced demyelinated corpus callosum, the density of Sox $2^{+}$cells was substantially increased during the time window of active oligodendrocyte regeneration (Hammond et al., 2015), for example, at 1 week after withdrawal of 6 week cuprizone treatment $(6+1$ week) as 
Table 2. Differentially expressed genes in the brain derived from RNA-seq ${ }^{a}$

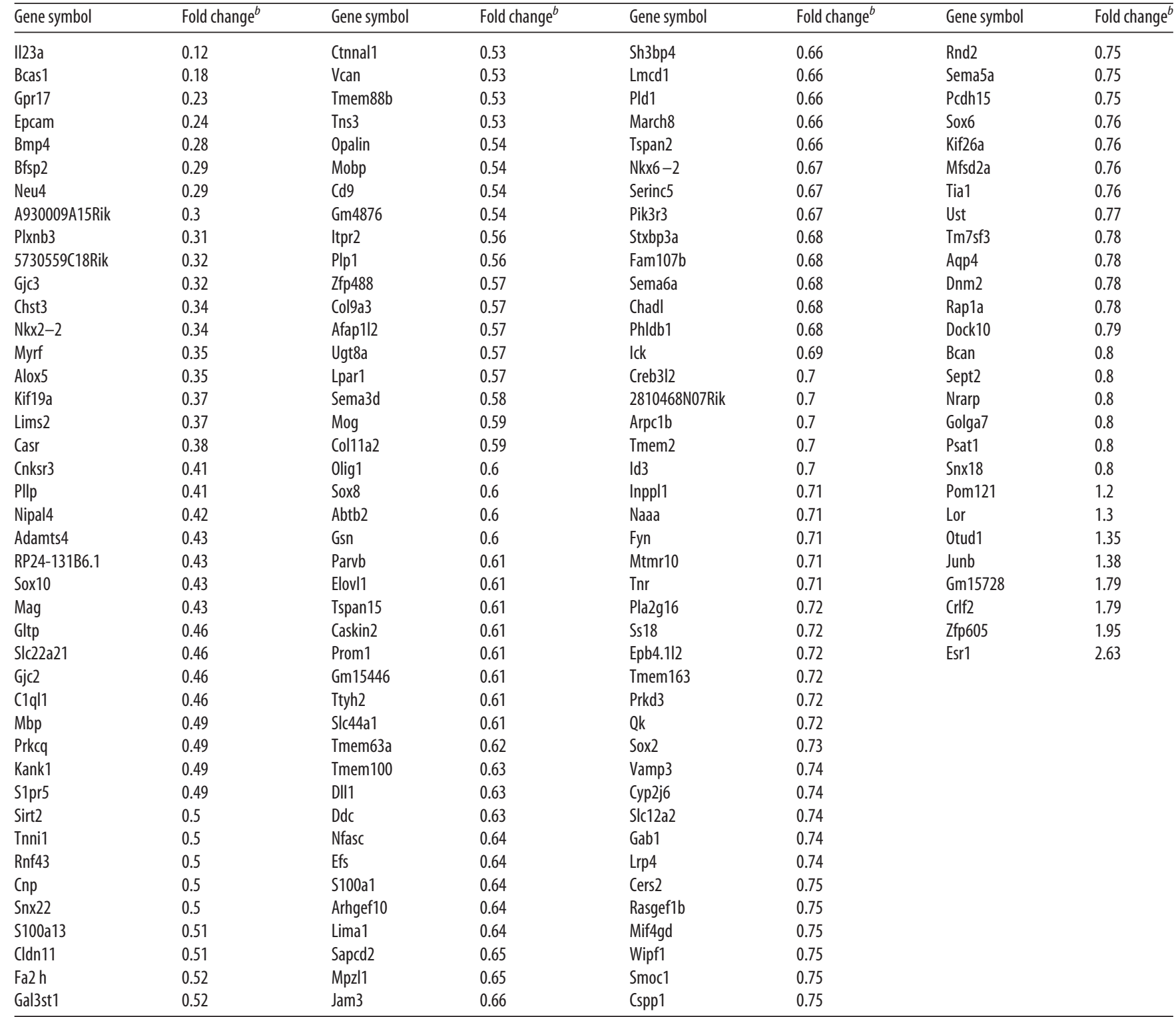

${ }^{a}$ Total RNAs are prepared from the whole forebrains of P14Pdgfr $\alpha$-CreER ${ }^{T 2}$, Sox ${ }^{\mathrm{fl} / \mathrm{fl}}(n=3)$ and Sox ${ }^{\mathrm{fl} / \mathrm{fl}}(n=3)$ mice that had received tamoxifen on P6 and P7. Only genes with a minimal change of 1.2 -fold and with a $p<0.05$ are shown. ${ }^{b}$ The ratio of the read counts in Sox2 CK0 to Sox 2 WT brains.

shown in Figure $8 B$, compared with the normal-chow controls (Fig. 8A). Consistent with the observations from developmental myelination (Fig. 1), Sox2 was also expressed in most TCF712 ${ }^{+}$ newly regenerated premyelinating OLs (Hammond et al., 2015) (Fig. 8C, arrowheads). Triple immunohistochemistry of Sox2, NG2, and TCF7l2 (Fig. 8D, top) showed that the level of Sox 2 in TCF7l2 ${ }^{+}$newly regenerated OLs was significantly higher than that in $\mathrm{NG}_{2}{ }^{+}$OPCs in the corpus callosum of mice maintaining on 6 consecutive weeks of $0.25 \%$ cuprizone diet (Fig. $8 D$, bottom). In the MOG-EAE spinal cord in which OPC proliferation and OL regeneration are consistently observed (Tripathi et al., 2010; Guo et al., 2011), more Sox ${ }^{+}$TCF712 $^{+}$newly regenerated OLs were observed (Fig. $8 F$, arrowheads) compared with scarce TCF7l2 $2^{+}$OLs in the CFA control spinal cord (Fig. 8E, arrowheads) at day 21 (D21) post-MOG immunization (Fig. 8G). Similarly, Sox2 expression levels in TCF712 ${ }^{+}$newly regenerated OLs were significantly higher than that in $\mathrm{NG}_{2}{ }^{+}$OPCs in the D21 spinal cord treated with MOG-peptide ${ }_{35-55}$ (Fig. $8 H, I$ ). These data suggest that Sox 2 may play a role in oligodendrocyte regeneration and remyelination during remyelination.

\section{Sox 2 is essential for brain remyelination after cuprizone-induced myelin damage}

Cuprizone-induced demyelination/remyelination in murine corpus callosum is a well-established animal model for studying molecular mechanisms underlying OL regeneration and myelin repair. Because constitutive Sox 2 cKO affected brain developmental myelination, we used the time-conditioned, tamoxifeninducible Pdgfr $\alpha$-CreER ${ }^{\mathrm{T} 2}$, Sox ${ }^{\mathrm{fl} / \mathrm{fl}}$ (Pdgfr $\alpha$-Sox $\left.2 \mathrm{cKO}\right)$ to study the role of Sox 2 in OL regeneration and remyelination.

Adult Pdgfr $\alpha$-Sox 2 cKO and non-Cre Sox $2^{\text {fl/fl }}$ control mice were intraperitoneally injected three times of 5 day course of tamoxifen and BrdU starting from the third week of cuprizone diet maintenance (for experimental designs, see Fig. 9A). Brain tissues were analyzed at the end of 1 week after returning to normal diet (Fig. 9A), a time point of active OL differentiation (Hammond et al., 2015). In the adult corpus 

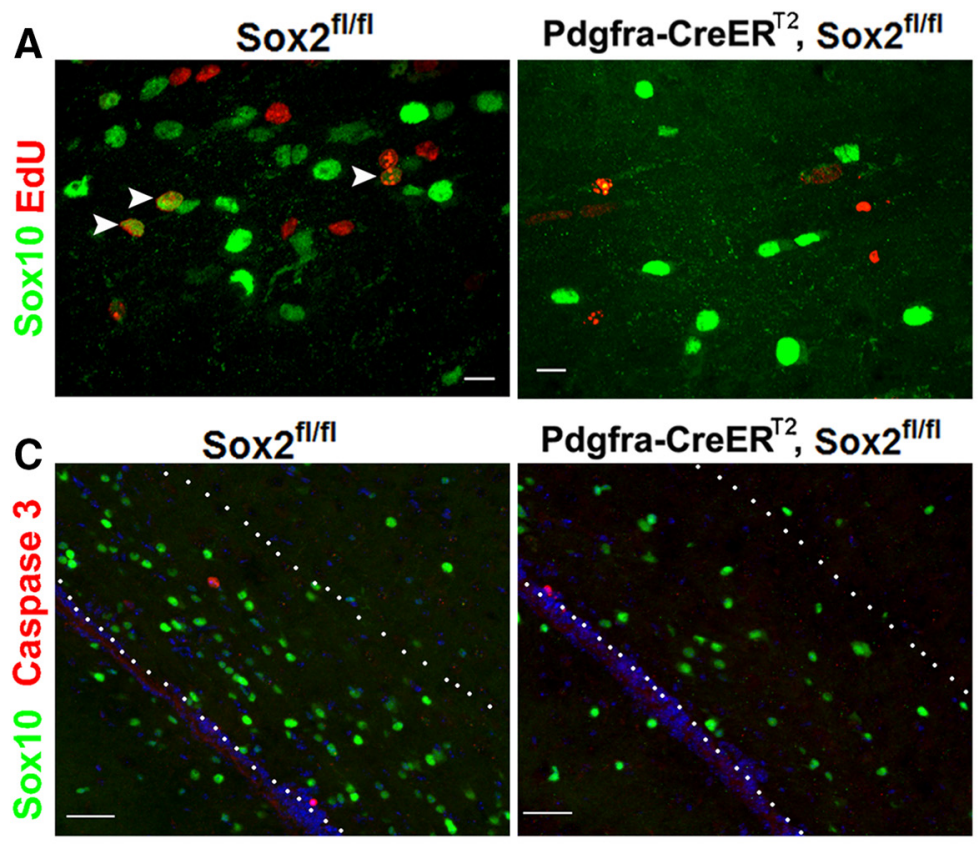

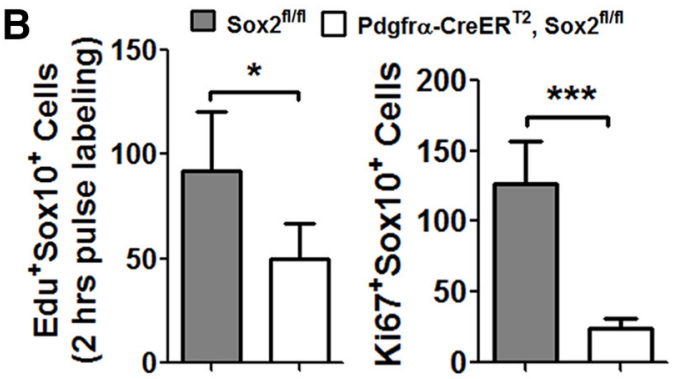

D

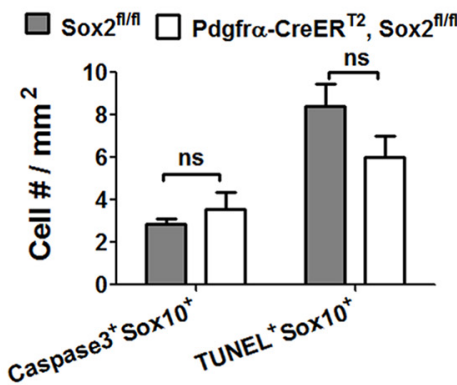

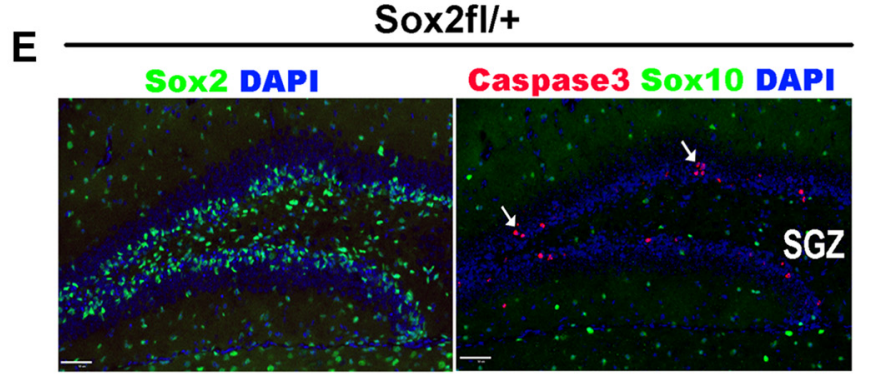

$\mathbf{F}$

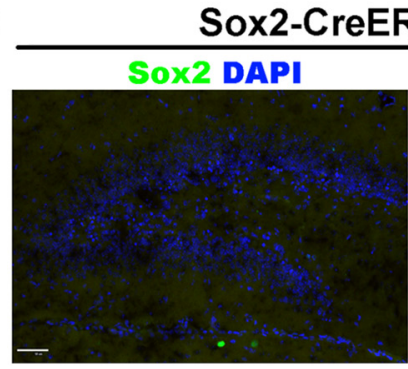

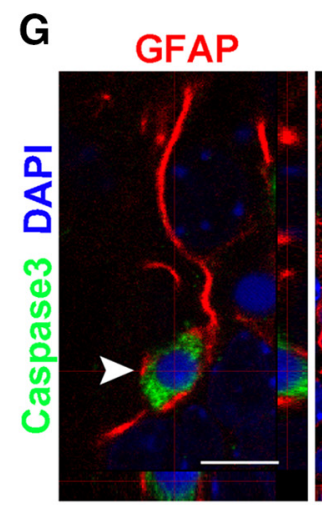
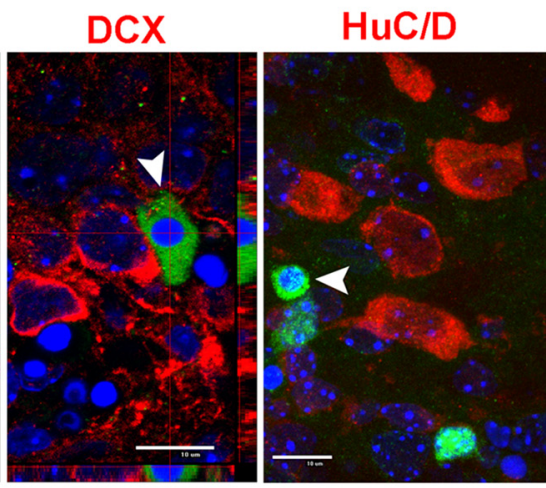

Figure 6. Sox2 regulates OPC proliferation but not survival. $A$, Confocal images of EdU ( $2 \mathrm{~h}$ of pulse labeling before death on P14) and Sox10 double immunostaining. B, Quantification (cell no. $/ \mathrm{mm}^{2}$ ) of EdU ${ }^{+}$Sox $10^{+}$and $\mathrm{Ki}^{+} 7^{+}$Sox $10^{+}$proliferative OPCs in the P14 forebrain subcortical white matter. C, Representative confocal images of Sox 10 and active caspase 3 double immunostaining in the P14 subcortical white matter (dotted areas). D, Quantification of Caspase ${ }^{+}$Sox $10^{+}$and TUNEL ${ }^{+}$Sox $10^{+}$oligodendroglial lineage cells in the P14 subcortical white matter. $\boldsymbol{A}-\boldsymbol{D}$, Animals are P14 old; tamoxifen treatment on P6 and P7. $N=3$ in each group. . ${ }^{*}<<0.05$ (two-tailed Student's $t$ test). ${ }^{* * *} p<0.001$ (two-tailed Student's $t$ test). $\boldsymbol{E}, \boldsymbol{F}$, Immunostaining of Sox2 (left panels), and active Caspase3 and Sox10 (right panels) in the neural stem cell niche of hippocampal SGZ of P14 non-Cre control mice (E) and Sox2-Sox2 cK0 mice (F) (both received tamoxifen on P6 and P7). Sox2 is completely removed from all Sox2-expressing cells, and active Caspase ${ }^{+}$cells are present and increased in the SGZ neural stem cell niche areas, but not in Sox10 ${ }^{+}$ oligodendroglial lineage cells in the Sox2-Sox2 cKO mice. $\boldsymbol{E}$, $\boldsymbol{F}$, Arrows point to representative active Caspase $3^{+}$cells. G, Double immunostaining showing that active Caspase $3^{+}$apoptotic cells (arrowheads) in the SGZ are GFAP ${ }^{+}$radial-like neural stem cells (left), but not doublecortin $(D C X)^{+}$neuroblasts, or HuC/D ${ }^{+}$neurons in the P14 Sox2-Sox2 cK0 mice that had received tamoxifen at P6 and P7. Scale bars: $A, 10 \mu \mathrm{m} ; C, 20 \mu \mathrm{m} ; \boldsymbol{E}, \boldsymbol{F}, 50 \mu \mathrm{m} ; \boldsymbol{G}, 10 \mu \mathrm{m}$.

callosum, all PDGFR $\alpha^{+}$OPCs express Sox 2 in Sox ${ }^{\text {fl/fl }}$ control mice (Fig. 9B1), and the tamoxifen paradigm used in the study (Fig. 9A) resulted in $\sim 90 \%$ efficiency of Sox 2 knock-out in OPCs. As shown in Figure 9B2, all EYFP-fate-mapped, PDGFR $\alpha^{+}$OPCs had no detectable Sox 2 expression (PDGFR $\alpha^{+}$Sox $2^{-} \mathrm{EYFP}^{+}$) in the corpus callosum of Pdgfr $\alpha$-CreER ${ }^{\mathrm{T} 2}$, Sox $2^{\mathrm{fl} / \mathrm{fl}}$, Rosa-EYFP triple transgenic mice.

One week after returning to normal diet, the densities of $\mathrm{CC}^{+}{ }^{+}$Olig ${ }^{+}$OLs (Fig. 9C) and PDGFR $\alpha^{+}$OPCs were signifi- cantly diminished in the corpus callosum of Pdgfr $\alpha$-Sox $2 \mathrm{cKO}$ mice compared with non-Cre control (Sox2 WT) mice (Fig. 9D). $\mathrm{BrdU}$ is incorporated into proliferative OPCs (but not postmitotic OLs) upon administration, and $\mathrm{CC}^{+}{ }^{+} \mathrm{BrdU}^{+}$OLs are representatives of newly regenerated OLs that inherited BrdU from $\mathrm{BrdU}^{+} \mathrm{OPCs}$ during the time window between BrdU treatment and tissue analysis. Our quantification data showed that $\mathrm{CC}^{+}{ }^{+} \mathrm{BrdU}^{+}$OLs were fewer in Pdgfr $\alpha$-Sox 2 cKO mice than that in non-Cre Sox $2^{\mathrm{fl} / \mathrm{fl}}$ controls (Fig. 9D). We also observed that 


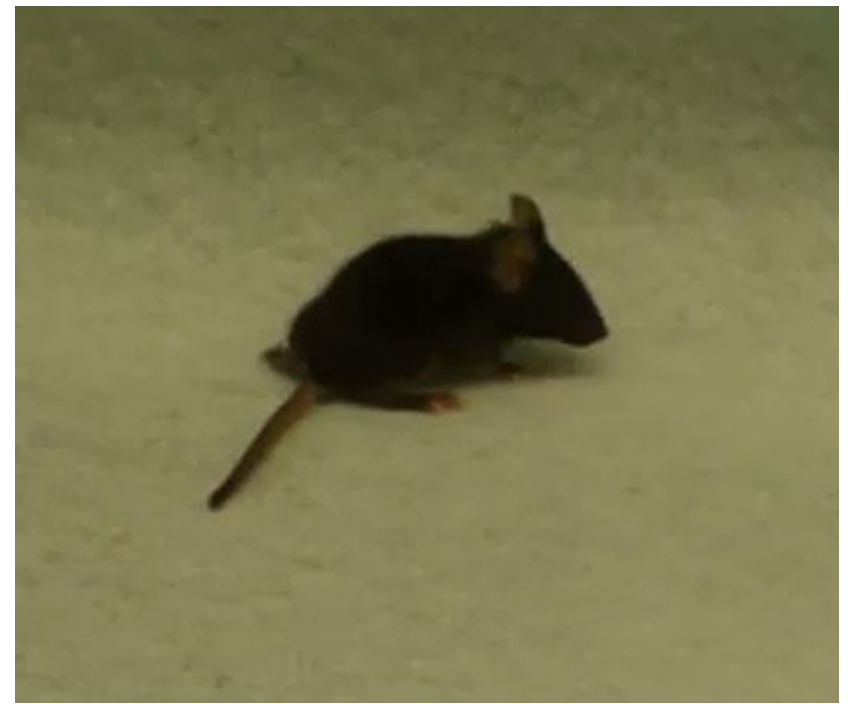

Movie 1. Presence of severe tremors and ataxia in Sox10-Sox2 cKO mice.

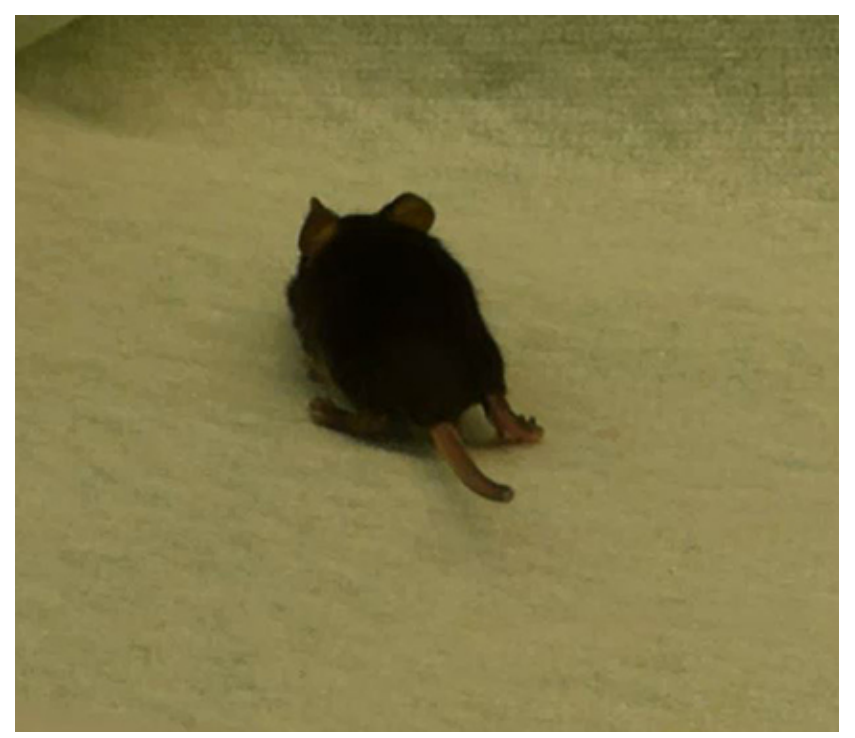

Movie 2. Absence of tremors and ataxia in Sox2 WT mice.

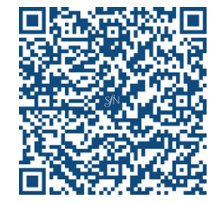

Pdgfr $\alpha$-Sox 2 cKO resulted in fewer Ki6 $7{ }^{+}$PDGFR $\alpha^{+}$proliferative OPCs (Fig. 9D). Using APC and TCF712 to label newly regenerated OLs (Fig. 9E) (Hammond et al., 2015), we found that the density of $\mathrm{APC}^{+} \mathrm{TCF}_{12}{ }^{+}$newly regenerated OLs was significantly lower in cuprizone-treated corpus callosum of Pdgfr $\alpha$ Sox2 cKO mice than that of non-Cre control mice (Fig. $9 F$ ). Immunohistochemistry of MBP and pan axonal marker SMI312 (Fig. $9 \mathrm{G}$ ) showed that $\mathrm{MBP}^{+}$myelin density was reduced by $50 \%$ in the Pdgfr $\alpha$-Sox 2 cKO corpus callosum, whereas SMI $312^{+}$axonal density was indistinguishable (Fig. 9H). Together, our data suggest that Sox 2 is required for remyelination in the adult corpus callosum after chemical-induced demyelination.

\section{Sox2 regulates OPC proliferation and OL regeneration in the spinal cord after inflammation-induced demyelination}

In the MOG-peptide ${ }_{35-55}$-induced EAE model, inflammationinduced demyelination in the spinal cord elicits a robust augment of OPC proliferation and a modest increase of oligodendrocyte regeneration (Tripathi et al., 2010), although the extent of remyelination is much less than that in cuprizone-induced demyelination model (Jones et al., 2008; Constantinescu et al., 2011). Therefore, we used MOG-peptide ${ }_{35-55}$-EAE animal model and Pdgfr $\alpha$-CreER ${ }^{T 2}$, Sox $2^{\mathrm{fl} / \mathrm{fl}}$ transgenic mice to study the role of Sox2 in regulating OPC proliferation and OL regeneration in the spinal cord after inflammation-induced demyelination.

We showed that all NG2 ${ }^{+}$OPCs in the adult spinal cord expressed Sox2 (Fig. 10B1). To avoid potential effects of Sox2 ablation before injury on OPC response to subsequent inflammation, we administered tamoxifen Pdgfr $\alpha$-Sox 2 cKO and non-Cre Sox $2^{\mathrm{fl} / \mathrm{fl}}$ (Sox2 WT) mice after massive CNS inflammation occurred, typically at day 9-12 after MOG immunization (Fig. $10 A)$. Sox 2 was ablated in virtually all NG2 ${ }^{+}$OPCs in the Pdgfr $\alpha$ Sox 2 cKO spinal cord (Fig. 10B3, arrowhead and boxed area), in sharp contrast to Sox2 WT spinal cord in which all NG2 ${ }^{+}$OPCs had Sox 2 expression (Fig. 10B2, arrowheads and boxed area). In agreement of our previous report (Guo et al., 2011), MOGpeptide immunization resulted in a fourfold increase in the density of Sox $10^{+} \mathrm{NG} 2{ }^{+}$OPCs (Fig. 10C1) in the lumbar spinal cord of Sox2 WT mice treated with MOG (Sox2 WT + MOG) compared with Sox2 WT mice treated with CFA control (Sox2 WT + CFA) (Fig. 10C3). However, Sox2 deletion significantly reduced the OPC density in the spinal cord of Pdgfr $\alpha$-Sox 2 cKO mice treated with MOG (Sox2 cKO + MOG) (Fig. 10C2,C3), indicating that Sox 2 is required for OPC population expansion after inflammatory insults. Notably, the similar distribution patterns of OPCs within inflammatory lesions were observed in the spinal cords between Pdgfr $\alpha$-Sox 2 cKO and Sox 2 WT mice with MOG (Fig. $10 B 2$ vs $B 3, C 1$ vs $C 2$ ), indicating that $S$ ox 2 appears dispensable for OPC recruitment into the inflammation-induced demyelination lesions. The number of $\mathrm{Ki} 67^{+} \mathrm{Olig} 2^{+}$proliferating OPCs (Fig. 10D1,D2) was significantly decreased in the spinal cord of Pdgfr $\alpha$-Sox 2 cKO mice treated with MOG (Sox 2 cKO + MOG) compared with Sox2 WT mice treated with MOG (Sox2 WT + MOG) (Fig. 10E).

Our previous study reports that the number of $\mathrm{TCF} 12^{+} \mathrm{APC}^{+}$ newly regenerated OLs increases in the spinal cord of MOG treatment (Fig. 10F) (Hammond et al., 2015). Nevertheless, Sox2 deletion resulted in significant decrease in the generation of $\mathrm{TCF}_{12}{ }^{+} \mathrm{APC}^{+} \mathrm{OLs}$ in the spinal cord of Pdgfr $\alpha$-Sox 2 cKO mutants treated with MOG (Fig. 10F). Together, these data suggest that Sox2 is essential for OPC proliferation and OL regeneration in the spinal cord after inflammation-induced demyelination.

\section{Discussion}

There are several novel findings in this study: (1) Sox2 is upregulated in newly differentiated OLs during developmental myelination and in newly regenerated OLs during remyelination; (2) Sox 2 is essential for brain developmental myelination by regulating OPC proliferation and OL differentiation; and (3) in the context of myelin repair, Sox2 is required for OPC proliferation and/or OL regeneration not only in autoimmune-induced spinal cord demyelination lesions but also in chemical-induced brain demyelination lesions. 
A
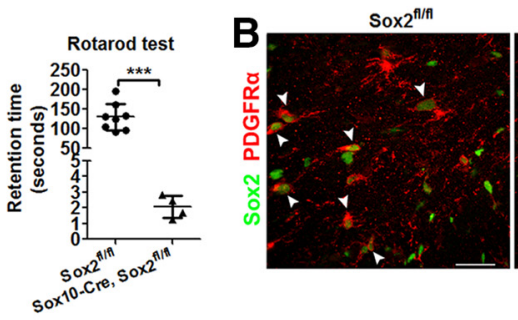
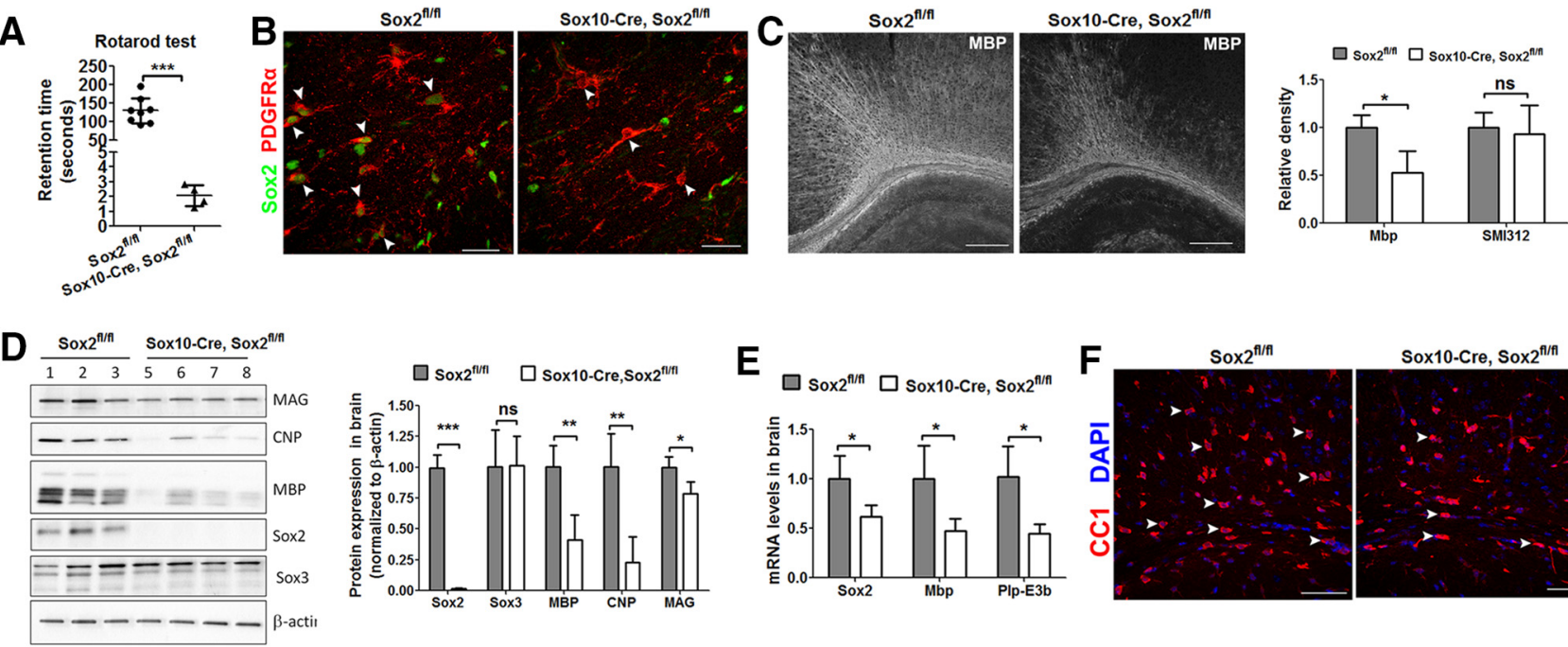

E
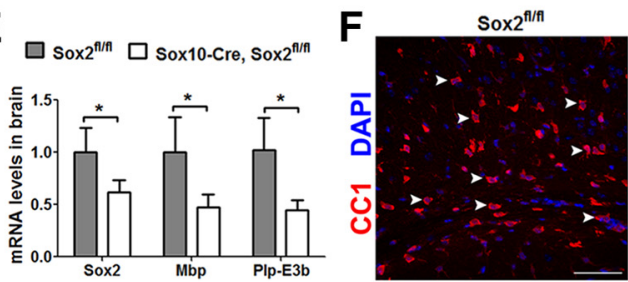

Sox10-Cre, Sox $2^{n / 1 / 1}$

G $\square \operatorname{sox} 2^{\mathrm{n} / \mathrm{f}}$

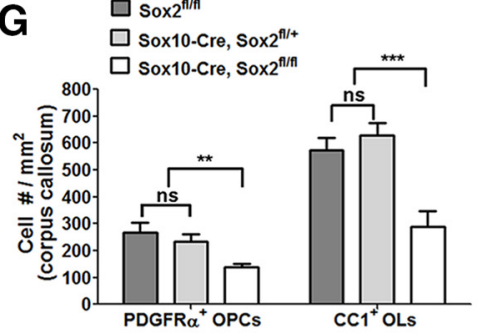

Sox $2^{\text {fl/f }}$

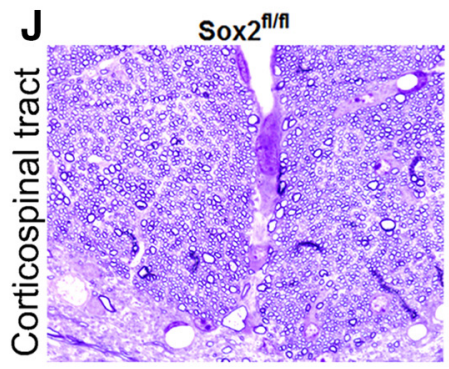

$\mathbf{H}$

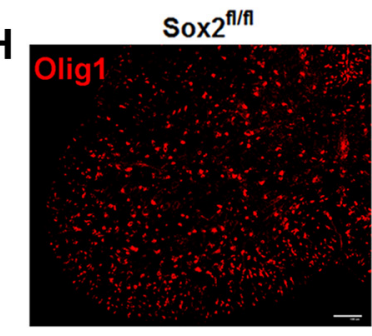

Sox10-Cre, Sox $2^{\text {fl/f }}$

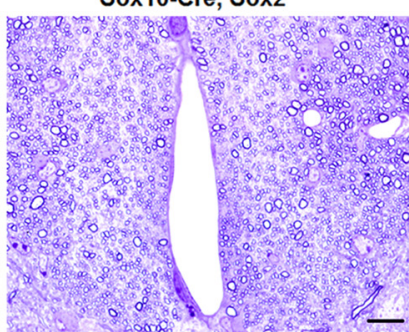

Sox10-Cre, So $2^{\text {fiff }}$

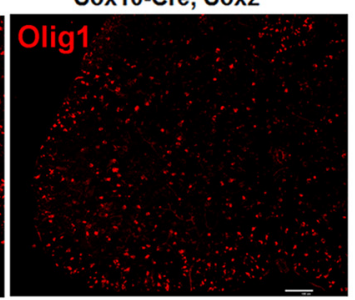

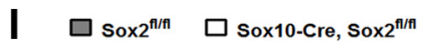
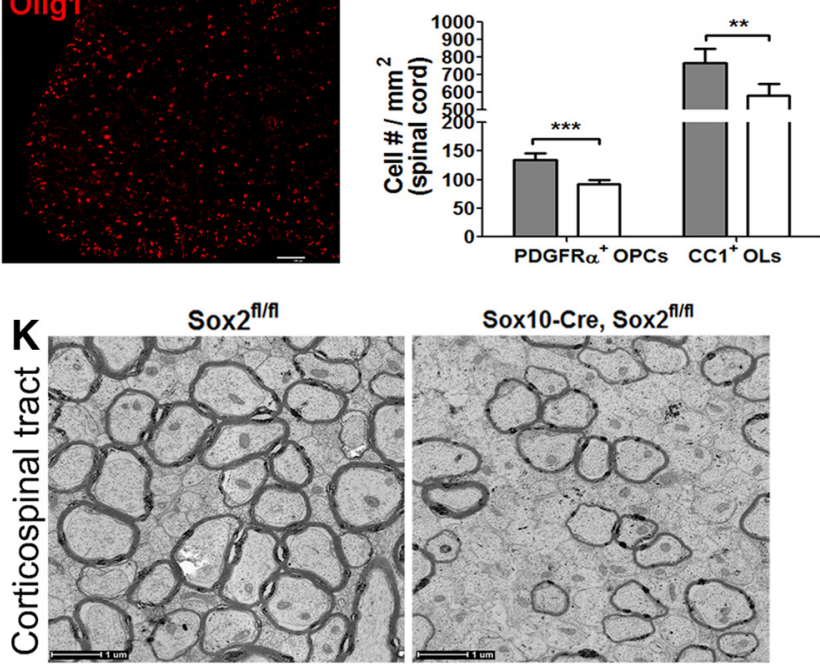

Figure 7. Oligodendrocyte differentiation and myelination are severely affected in the Sox2 CKO brain at later stages of postnatal development. $A$, Accelerating Rotarod test showing that Sox10-Sox2 cKO (Sox10-Cre, Sox $2^{\mathrm{fl} / \mathrm{fl})}$ mice have severely impaired motor performance function evidenced by significant less retention time on the rotating rod ( $n=8$ Sox ${ }^{\mathrm{fl} / \mathrm{ff}}, n=4$ Sox 10 -Sox2 cK0, P28). B, Double immunostaining of Sox2 and PDGFR $\alpha$ in the corpus callosum of Sox10-Sox2 cK0 and control mice. Sox 2 is expressed in OPCs of the control mice (arrowheads, left) and absent from OPCs in the Sox10-Sox2 cK0 mice (arrowheads, right). C, Grayscale images showing decreased MBP ${ }^{+}$myelin fibers in the corpus callosum and overlaying cingular cortex and quantification of MBP and SMI312 densities $\left(n=4\right.$ Sox10-Sox2 cK0, $n=5$ Sox2 $\left.{ }^{\mathrm{fl} / f l}\right)$. D, Western blotting of Sox2, another SoxB1 family member Sox3, myelin-related genes MBP, CNP, and MAG (left), and quantifications (right). The protein levels of MBP and CNP are reduced by $>50 \%$ in P21 Sox10-Sox2 cK0 brains, compared with non-Cre controls. E, qRT-PCR of forebrain mRNA levels of Sox2, MBP, and myelinating 0L-specific PIp-Exon3b ( $n=4$ Sox10-Sox2 CK0, $n=6$ non-Cre controls, P21). $F$, Immunostaining of mature $0 \mathrm{~L}$ marker CC1 in the P21 corpus callosum. Arrowheads point to representative $\mathrm{CC}^{+}{ }^{+}$OLs. G, Quantification of PDGFR $\alpha^{+}$OPCs and CC $1^{+}$OLs in forebrain subcortical white matter tracts $(n=4$ Sox 10-Sox2 cK0, $n=5$ Sox 10-Sox2 hetero-cK0, and $n=7$ non-Cre controls, P21). H, Low-magnification images showing similar distribution but decreased density of Olig1-expressing oligodendroglial lineage cells in the P21 spinal cord of Sox10-Sox2 cKO (right) compared with Sox2 ${ }^{\mathrm{f} / / \mathrm{fl}}$ control (left). I, Quantification of PDGFR $\alpha^{+}$OPCs and CC ${ }^{+}$OLs in the P21 spinal cord ( $n=6$ Sox2 $2^{\mathrm{fl} / f l}, n=4$ Sox10-Sox2 cKO). J, $\boldsymbol{K}$, Corticospinal tract cross semithin sections of toluidine blue staining $(\boldsymbol{J})$ and ultrathin sections of transmission electron microscopy $(\boldsymbol{K})$ from a P28 Sox10-Sox2 mouse and a littermate Sox $2^{\mathrm{f} / \mathrm{fl}}$ control. The density of myelinated axons is substantially decreased at low $(\boldsymbol{J})$ and high $(\boldsymbol{K})$ magnification images. Two-tailed Student's $t$ test in $\boldsymbol{A}, \boldsymbol{C}, \boldsymbol{D}, \boldsymbol{E}$, I, one-way ANOCA with Tukey's post hoc test in $\boldsymbol{G}:{ }^{*} p<0.05$; ${ }^{* *} p<0.01$; ${ }^{* * *} p<0.001$. Scale bars: $\boldsymbol{B}, 25 \mu \mathrm{m} ; \boldsymbol{C}, 200 \mu \mathrm{m} ; \boldsymbol{F}, 50 \mu \mathrm{m} ; \boldsymbol{H}, 100 \mu \mathrm{m} ; \boldsymbol{J}, 10 \mu \mathrm{m} ; \boldsymbol{K}, 1 \mu \mathrm{m}$.

In this study, we found that OPCs retain Sox2 expression in the adult murine CNS. Previous study reports that Sox $2^{+} \mathrm{Olig} 2^{+}$ cells (presumably Sox $2^{+}$OPCs) can be immunohistochemically detected in the adult human white matter and that these Sox $2 /$ Olig2-positive cells can be differentiated into mature oligodendrocytes in vitro (Oliver-De La Cruz et al., 2014). This report, together with our finding in the murine CNS, suggests that Sox2 expression in adult OPCs is well conserved from rodent to human CNS. However, the function of Sox2 in adult OPCs under normal conditions has not been defined. Our study demonstrates that Sox2 is required for OPC proliferation and population ex- pansion during developmental myelination (Fig. 5A-F). Inspired by these data, we hypothesize that Sox 2 is required for adult OPC homeostasis and brain myelin turnover and/or remodeling (Zhang et al., 1999; Yeung et al., 2014) and is essential for OPCs' response to various demyelinating insults, the latter of which is in agreement with a recent report showing that Sox 2 deletion before lysolecithin-induced focal demyelination reduces progenitor response and recruitment to demyelinating lesions (Zhao et al., 2015).

Interestingly, we also found that Sox 2 is upregulated in a subpopulation of differentiated OLs during developmental myelina- 

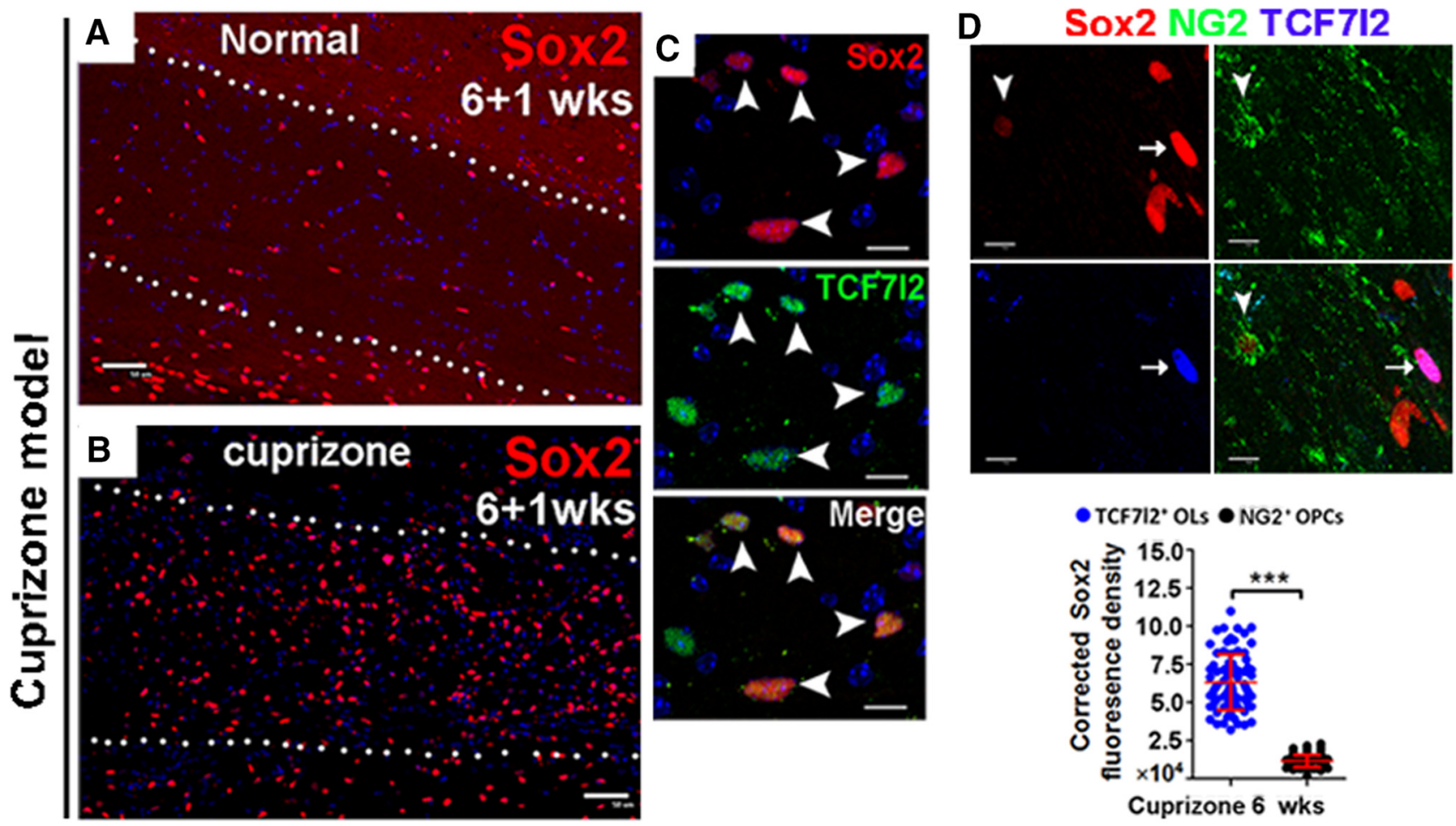

- TCF712* OLS • NG2*OPCS


Figure 8. Sox 2 is expressed in adult $\mathrm{OPCS}$ and upregulated in TCF7l2-expressing $0 \mathrm{Ls}$ in the spinal cord and brain during remyelination. $A, B$, Low-magnification confocal images showing Sox2 expression in the corpus callosum of adult mice fed with normal diet $(\boldsymbol{A})$ and with 6 weeks of $0.25 \%$ cuprizone diet followed by 1 week normal diet $(6+1$ weeks) (B). Dotted areas delineate corpus callosum in the mid-sagittal brain sections. C, High-power confocal images showing Sox2 expression in TCF712 ${ }^{+}$newly regenerated 0Ls at $6+1$ weeks. D, Triple immunostaining of Sox2, NG2, and TCF7I2 (top) and quantification (bottom) in the corpus callosum of adult mice fed with 6 weeks of cuprizone diet. $\boldsymbol{E}-\mathbf{G}$, Confocal images showing Sox2 expression in TCF7I2 ${ }^{+} 0$ Ls in the lumbar spinal

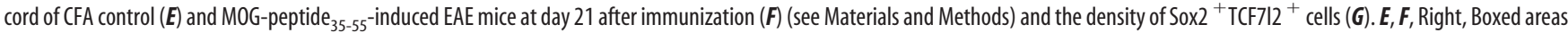
represent higher magnification. $\boldsymbol{H}, \boldsymbol{I}$, Triple immunostaining of Sox2, NG2, and TCF7I2 (top) and quantification (bottom) in the lumbar spinal cord of adult mice at $21 \mathrm{~d}$ (D21) after immunization, and the corrected Sox2 fluorescence density of TCF712 ${ }^{+} 0 \mathrm{Ls}$ and NG2 ${ }^{+} \mathrm{OPCS} .{ }^{* *} p<0.001$. Scale bars: $A, B, E, F, 50 \mu \mathrm{m} ; C, D, H, 10 \mu \mathrm{m}$. 
A Pdgfr $\alpha$-CreER ${ }^{T 2}$, So $\times 2^{f 1 / f l}$, Rosa-EYFP mice

\begin{tabular}{|c|c|c|c|c|c|c|}
\hline So $\times 2^{f 1 / f 1}$ & $-E\rangle$ & & TM+BrdU & 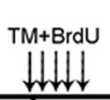 & $\begin{array}{c}{ }^{T M}+\mathrm{BrdU} \\
\text { HWt }\end{array}$ & ${ }_{\uparrow}^{\text {Tissue analysis }}$ \\
\hline eek 0 & 1 & 2 & 3 & 4 & 5 & 7 \\
\hline & \multicolumn{5}{|c|}{ cuprizone diet } & normal diet \\
\hline
\end{tabular}

B1 Sox $2^{\mathrm{fl} / \mathrm{fl}}$

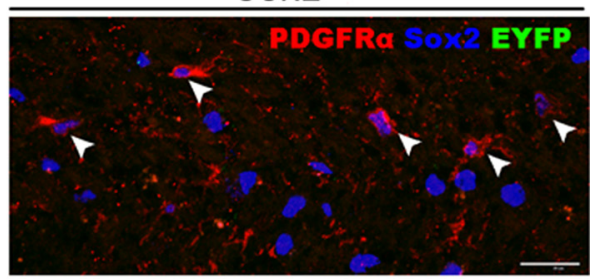

B2 Pdgfro-CreER ${ }^{T 2}$, Sox $2^{\mathrm{fl} / \mathrm{fl}}$

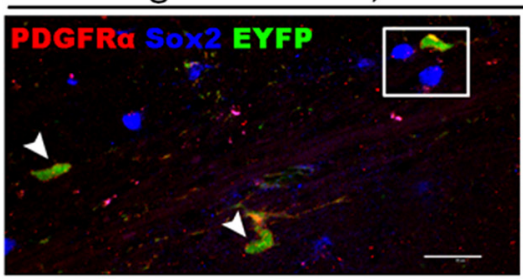

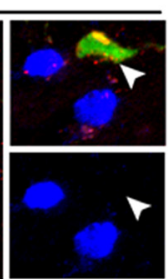

B3

Sox2 WT

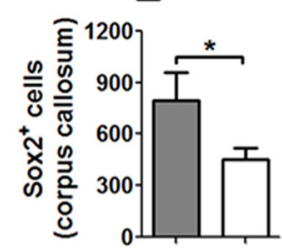

C

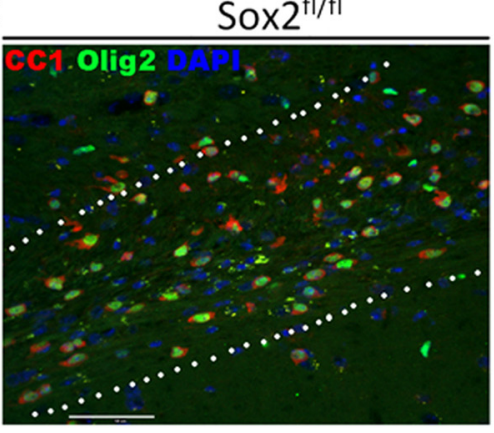

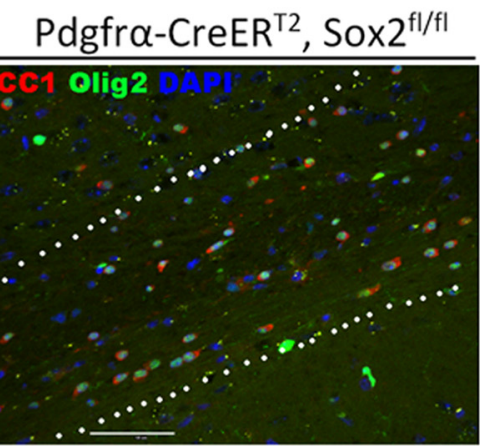

D

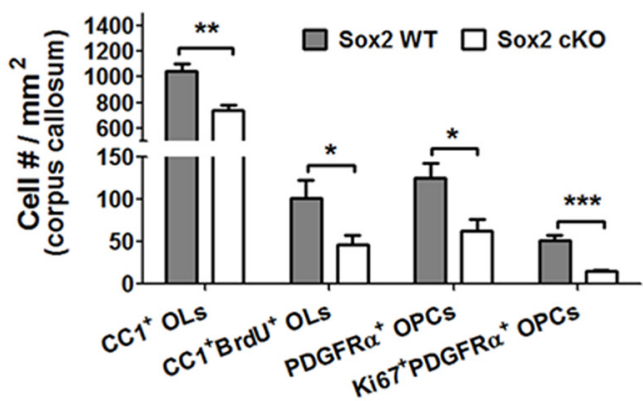

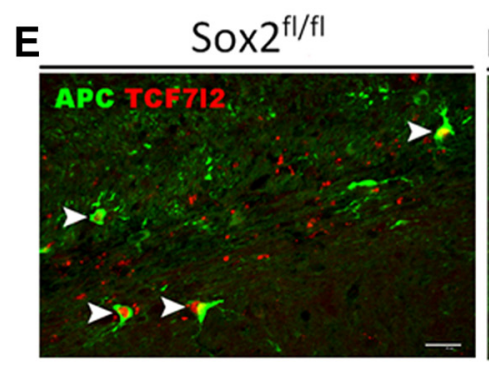

G

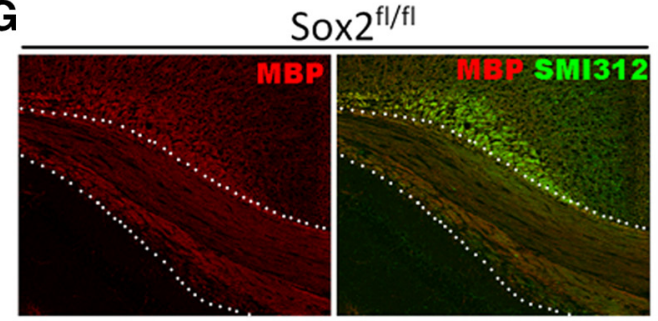

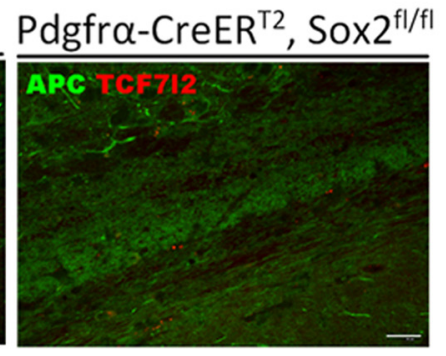

$\mathbf{F}$

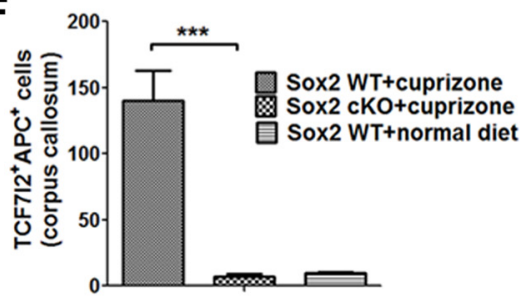

Figure 9. Sox2 is required for $0 \mathrm{~L}$ regeneration and remyelination in cuprizone-induced demyelinated corpus callosum. $\boldsymbol{A}$, Transgenic mice (male, 2-3 months old at onset of cuprizone diet) and experimental designs for $\boldsymbol{B} 1-\boldsymbol{H}$. The $0.25 \%$ curprizone diet was used in the design. B1, B2, Representative confocal images of Sox2, PDGFR $\alpha$, and EYFP triple immunostaining in corpus callosum. Arrowheads point to PDGFR $\alpha{ }^{+}$Sox2 ${ }^{+}$EYFP $^{-}$cells (left) and PDGFR $\alpha{ }^{+}$Sox2 ${ }^{-}$EYFP ${ }^{+}$cells (right). B2, Right, Boxed area represents higher-magnification images. B3, Density of Sox2 ${ }^{+}$cells (cell no. $/ \mathrm{mm}^{2}$ ) in corpus callosum ( $n=3$ each group). C, Double immunostaining of (C1 and Olig2. Dotted areas represent the posterior corpus callosum in mid-sagittal forebrain sections, which is consistently affected in the cuprizone model (Steelman et al., 2012). D, Densities of marker-positive cell as indicated ( $n=3$ each group). $\boldsymbol{E}$, Representative confocal images of APC and TCF7l2 double immunostaining. Arrowheads point to APC ${ }^{+}$TCF7I2 ${ }^{+}$newly regenerated OLs (Lang et al., 2013; Hammond et al., 2015). F, Quantification of APC ${ }^{+}$TCF7I2 ${ }^{+}$cells ( $n=3$, Sox2 WT + cuprizone; $n=$ 3, Sox2 cKO + cuprizone; $n=4$ Sox2 WT + normal diet). G, Confocal images showing MBP and SMI312 in the posterior corpus callosum (coronal forebrain sections). $\boldsymbol{H}$, Quantification of MBP and SMI312 immunoreactive signals in $\boldsymbol{H}$ ( $n=3$ each group). $\boldsymbol{B 3}, \boldsymbol{D}, \boldsymbol{F}, \boldsymbol{H},{ }^{*} p<0.05$ (two-tailed Student's $t$ test). ${ }^{* *} p<0.01$ (two-tailed Student's $t$ test). ${ }^{* * *} p<0.001$ (tw0-tailed Student's $t$ test). Scale bars: $\boldsymbol{B} 1, \boldsymbol{B} \mathbf{2}, \boldsymbol{E}, 20 \mu \mathrm{m} ; \boldsymbol{C}, 50 \mu \mathrm{m} ; \boldsymbol{G}, 100 \mu \mathrm{m}$.

tion and remyelination in the rodent CNS. This upregulation is counterintuitive to the established concept that Sox2 maintains the "stemness" of neural stem/progenitor cells and is downregulated upon progenitor differentiation (Graham et al., 2003). Spe- cifically, Sox2 is transiently upregulated in the differentiated oligodendrocytes that are TCF712-positive, which we have previously identified as postmitotic, newly formed (regenerated) OLs (Hammond et al., 2015). Although the number of Sox $2^{+}$differ- 
A Pdgfra-CreER ${ }^{T 2}$, Sox2 $2^{f / f l}$ mice (Sox2 cKO)
Sox2 ${ }^{f / f l}$ mice (Sox2 WT)

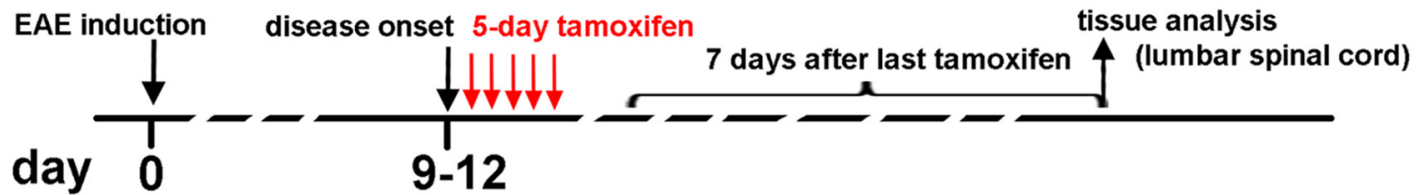

B1 Sox2 WT + CFA

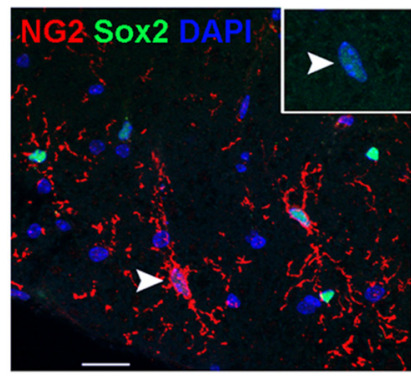

C1

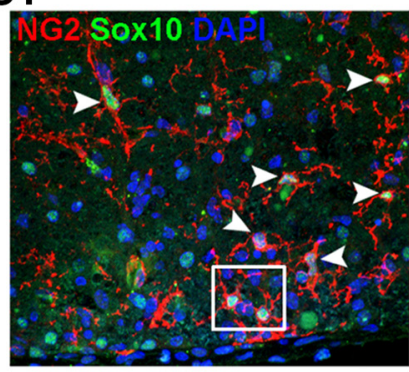

D1

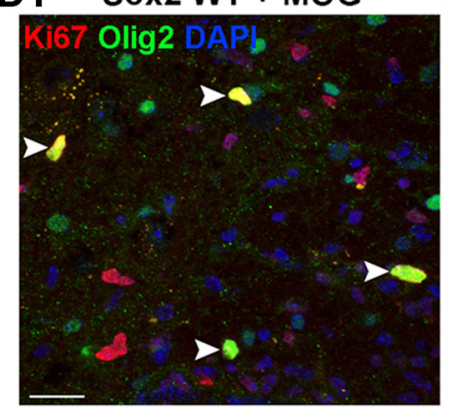

B2

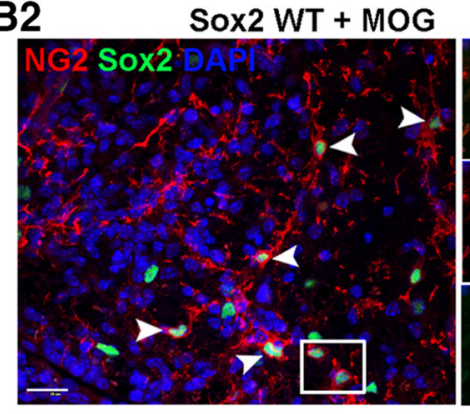

C2
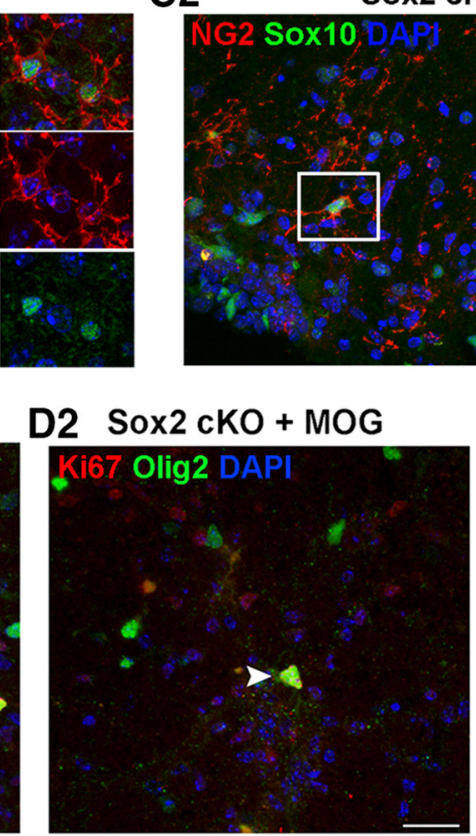

E

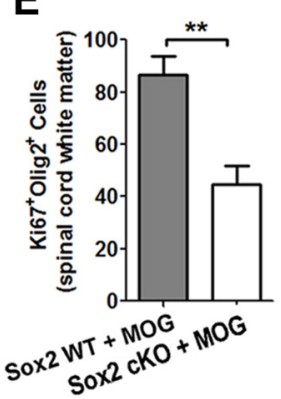

B3
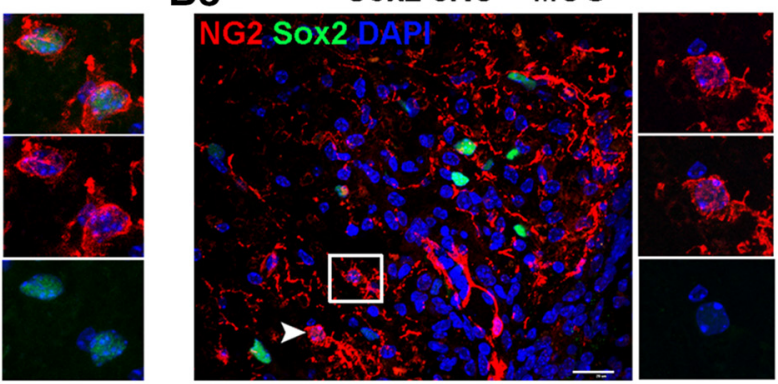

C3
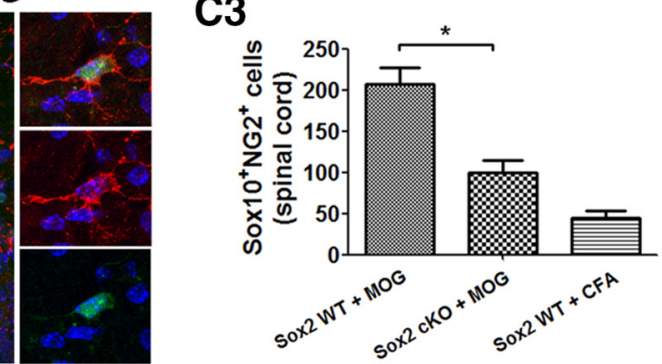

F

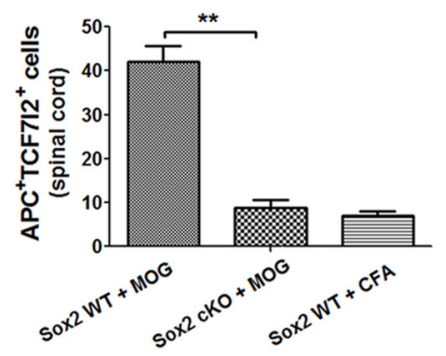

Figure 10. Sox2 regulates $\mathrm{OPC}$ proliferation and $0 \mathrm{~L}$ regeneration in the spinal cord in response to inflammatory insults. $A$, Transgenic mice (female, $2-3$ months old) and experimental designs for B1-F. EAE was induced at day 0 by immunizing study mice with MOG peptide ${ }_{35-55}$ emulsified in CFA (see Materials and Methods) (Guo et al., 2012). Seven days after the last tamoxifen injection, lumbar spinal cord was harvested for analysis. B1-B3, Double immunostaining of Sox2 and NG2 in the lumbar spinal cords of each group. Right, Boxed areas represent higher power. B2, B3, Demyelination lesions with extensive inflammatory infiltrations indicated by dense DAPI nuclear counterstaining. $B 1, B 2$, Arrowheads point to Sox2 ${ }^{+} \mathrm{NG} 2{ }^{+} \mathrm{OPCS}$. NG2 ${ }^{+} \mathrm{OPCS}$ in Sox2 $\mathrm{CKO}$ have no Sox2 expression (B3) but distribute within the demyelination lesions in a similar pattern to that in Sox2 WT mice (B2). C1-C3, Confocal images of NG2 and Sox10 double immunostaining (C1, C2) and quantification of Sox $10{ }^{+} \mathrm{NG2}{ }^{+} \mathrm{OPCS}(C 3)$. Arrowheads point to NG2 ${ }^{+} \mathrm{S} 0 \times 10^{+} \mathrm{OPCs}$. Right, Boxed areas represent higher-magnification images. D1-E, Representative confocal images of Ki67 and 0 lig2 $(\boldsymbol{D} 1, \mathbf{D 2})$ and quantifications $(\boldsymbol{E}) . \boldsymbol{F}$, Density of APC ${ }^{+}$TCF712 ${ }^{+}$newly regenerated $0 \mathrm{Ls} . N=3$ in each group. ${ }^{*} p<0.05$ (two-tailed Student's $t$ test). ${ }^{* *} p<0.01$ (two-tailed Student's $t$ test). Scale bars, $20 \mu \mathrm{m}$.

entiated OLs are reduced over time during developmental myelination, the percentage of Sox $2^{+}$cells among TCF712 ${ }^{+}$newly formed OLs did not change $(>90 \%$ at all time points we assessed). Given the asynchronous properties of in vivo OL differentiation, our quantitative data suggest that all OLs express Sox2 at certain developmental stages during OL lineage progression. Sox 2 upregulation is necessary for OL lineage progression and maturation, as Cnp-Sox $2 \mathrm{cKO}$ resulted in diminished myelin formation (Fig. 2) and OL differentiation (Fig. 3) in the brain. From a pretranslational perspective, it would be very important and interesting to test whether dose-controlled, transient Sox 2 overexpression in postmitotic OPCs and/or differentiating OLs is sufficient to promote OL differentiation and/or (re)myelination.

Our study suggests a previous unappreciated concept that Sox2 regulates CNS myelin formation and repair in CNS region and context-dependent manners. Using oligodendroglial-specific Sox2 cKO mice, Hoffmann et al. (2014) demonstrate that Sox2 is dispensable for OPC proliferation and migration; instead, it is required for OL differentiation in the embryonic and perinatal spinal cord (P3). 
More recently, Zhao et al. (2015) used the chicken $\beta$-actin promoter and CMV enhancer-driven Cre to ubiquitously ablate Sox2 (Cag$\mathrm{CreER}^{\mathrm{T} 2}$, Sox $2^{\mathrm{f} / \mathrm{fl}}$ ) and found that Sox 2 plays a minor, if any, role in OL differentiation and myelination in the postnatal spinal cord. In our study, we used Cnp-Cre to conditionally ablate Sox2 in the later stages of OL development (Dugas et al., 2010; Moyon et al., 2016; Zhao et al., 2016) and found a CNS region-dependent role of Sox 2 in regulating OL differentiation: it is required for brain OL differentiation (Fig. 3) and appears to play a minor role in OL differentiation (or regulate the timing of OL differentiation) during developmental myelination in the postnatal spinal cord (Fig. 4). The mechanisms underlying the CNS region-dependent role of Sox2 are unclear. Previous study shows that another SoxB1 family member Sox 3 functions redundantly with Sox2 in regulating spinal cord OL differentiation (Hoffmann et al., 2014). It is plausible that Sox3 may compensate the loss of Sox 2 during postnatal spinal cord development. However, the compensatory effects of Sox 3 on Sox 2 loss of function are less likely to occur in the brain myelination, as Sox10Cre, Sox $2^{\mathrm{f} / \mathrm{fl}}$ mutant mice display severe defects in motor function, OL differentiation, and brain developmental myelination (Fig. 7). Our results also indicate that, in the context of remyelination, Sox 2 is required for myelin repair in both adult brain (Fig. 9) and spinal cord (Fig. 10) through regulating OPC population supply and/or OL differentiation. Our remyelination study did not provide definitive evidence that Sox 2 is required for remyelination by directly regulating OL differentiation, as it does in brain myelination. In this regard, time-conditioned, stage-specific Sox 2 cKO or conditional overexpression paradigms are needed to unequivocally define the role of Sox 2 in regulating OL differentiation itself after myelin damage.

Oligodendrocyte number was not affected in the spinal cord of the Cnp-Sox 2 cKO mutants at P14 (Fig. 4) but was impaired in the spinal cord of the Sox10-Sox $2 \mathrm{cKO}$ mutants even at later ages (Fig. $7 H-K)$. This discrepancy presumably reflects the stagespecific Sox 2 cKO in oligodendroglial lineage cells. In the Sox10Sox 2 cKO mutants, Sox2 was initially ablated in the upstream proliferating OPCs, whereas in the Cnp-Sox 2 cKO mice, primarily in the differentiation-committed, late-stage OPCs and OLs. The reduced number of differentiated OL observed in Sox10Sox 2 cKO spinal cord is likely due to the reduced proliferation and supply of OPCs as we noticed diminished density of OPCs in the Sox10-Sox 2 cKO (but not in Cnp-Sox2 cKO at P14) spinal cord. These data are in line with our conclusion that Sox2 is required for OPC proliferation and population expansion.

The working model in which Sox 2 coordinates OPC proliferation and OL differentiation is compatible with the one derived from NSPCs (Episkopou, 2005) and with the expression patterns of Sox 2 along the progression of oligodendroglial lineage (Fig. $1 M)$. Previous studies have documented that Sox2 genetic knock-out (or knockdown) diminishes the proliferation of NSPCs under self-renewal conditions and reduces neuronal differentiation from NSPCs under neurogenic conditions (Ferri et al., 2004; Cimadamore et al., 2013). Mechanistically, Sox2 interacts with Chd7, a chromatin remodeler to directly regulate a variety of downstream signaling pathways, including Shh and Notch signaling pathways in NSPCs (Engelen et al., 2011), both of which are important regulators of oligodendroglial development (He and Lu, 2013; Wheeler and Fuss, 2016). Interestingly, a recent study shows that Chd7 regulates the onset of CNS myelination and remyelination through interacting with Sox10 (He et al., 2016). Biochemical evidence suggests that Sox 2 interacts with Sox10 to regulate Schwann cell development in the peripheral nervous system (Arter and Wegner, 2015). Our unpublished data show that Sox 2 is colabeled with Chd7 in oligodendroglial lineage cells at the histological level and displays similar expression dynamics to that of Chd7 (He et al., 2016). Therefore, it is tempting to hypothesize that Sox 2 may regulate brain developmental myelination and CNS remyelination through binding to Chd7Sox10 and/or through the downstream signaling pathways targeted by the putative Sox2-Chd7-Sox10 complex. Further studies are needed to support or falsify this hypothesis.

In conclusion, a series of genetic experiments in our study demonstrate that Sox2 plays a crucial role in regulating OPC proliferation and OL differentiation during postnatal brain development, although it appears to play a minor, transient role in OL differentiation during postnatal spinal cord development. Furthermore, we show that Sox 2 is required for myelin repair by regulating OPC proliferation and/or OL differentiation in both the demyelinated spinal cord and brain, suggesting a common mechanism. Our study suggests that Sox 2 may be a therapeutic target that can be experimentally manipulated to promote $\mathrm{OL}$ regeneration and CNS remyelination. Considering the established protocols of expressing Sox 2 in the stem cell biology field, it would be interesting to investigate whether Sox2 expression through genetic and/or viral-mediated approaches is sufficient to promote endogenous CNS remyelination after myelin damage.

\section{References}

Arter J, Wegner M (2015) Transcription factors Sox10 and Sox 2 functionally interact with positive transcription elongation factor $\mathrm{b}$ in Schwann cells. J Neurochem 132:384-393. CrossRef Medline

Bin JM, Harris SN, Kennedy TE (2016) The oligodendrocyte-specific antibody 'CC1' binds quaking 7. J Neurochem 139:181-186. CrossRef Medline

Cimadamore F, Amador-Arjona A, Chen C, Huang CT, Terskikh AV (2013) SOX2-LIN28/let-7 pathway regulates proliferation and neurogenesis in neural precursors. Proc Natl Acad Sci U S A 110:E3017-E3026. CrossRef Medline

Constantinescu CS, Farooqi N, O’Brien K, Gran B (2011) Experimental autoimmune encephalomyelitis (EAE) as a model for multiple sclerosis (MS). Br J Pharmacol 164:1079-1106. CrossRef Medline

Dai J, Bercury KK, Ahrendsen JT, Macklin WB (2015) Olig1 function is required for oligodendrocyte differentiation in the mouse brain. J Neurosci 35:4386-4402. CrossRef Medline

Dugas JC, Cuellar TL, Scholze A, Ason B, Ibrahim A, Emery B, Zamanian JL, Foo LC, McManus MT, Barres BA (2010) Dicerl and miR-219 are required for normal oligodendrocyte differentiation and myelination. Neuron 65:597-611. CrossRef Medline

Engelen E, Akinci U, Bryne JC, Hou J, Gontan C, Moen M, Szumska D, Kockx C, van Ijcken W, Dekkers DH, Demmers J, Rijkers EJ, Bhattacharya S, Philipsen S, Pevny LH, Grosveld FG, Rottier RJ, Lenhard B, Poot RA (2011) Sox 2 cooperates with Chd7 to regulate genes that are mutated in human syndromes. Nat Genet 43:607-611. CrossRef Medline

Episkopou V (2005) SOX2 functions in adult neural stem cells. Trends Neurosci 28:219-221. CrossRef Medline

Favaro R, Valotta M, Ferri AL, Latorre E, Mariani J, Giachino C, Lancini C, Tosetti V, Ottolenghi S, Taylor V, Nicolis SK (2009) Hippocampal development and neural stem cell maintenance require Sox2-dependent regulation of shh. Nat Neurosci 12:1248-1256. CrossRef Medline

Feng R, Zhou S, Liu Y, Song D, Luan Z, Dai X, Li Y, Tang N, Wen J, Li L (2013) Sox2 protects neural stem cells from apoptosis via up-regulating survivin expression. Biochem J 450:459-468. CrossRef Medline

Ferri AL, Cavallaro M, Braida D, Di Cristofano A, Canta A, Vezzani A, Ottolenghi S, Pandolfi PP, Sala M, DeBiasi S, Nicolis SK (2004) Sox2 deficiency causes neurodegeneration and impaired neurogenesis in the adult mouse brain. Development 131:3805-3819. CrossRef Medline

Graham V, Khudyakov J, Ellis P, Pevny L (2003) SOX2 functions to maintain neural progenitor identity. Neuron 39:749-765. CrossRef Medline

Guo F, Bannerman P, Mills Ko E, Miers L, Xu J, Burns T, Li S, Freeman E, McDonough JA, Pleasure D (2015) Ablating N-acetylaspartate prevents leukodystrophy in a Canavan disease model. Ann Neurol 77:884-888. CrossRef Medline

Guo F, Maeda Y, Ma J, Delgado M, Sohn J, Miers L, Ko EM, Bannerman P, Xu 
J, Wang Y, Zhou C, Takebayashi H, Pleasure D (2011) Macroglial plasticity and the origins of reactive astroglia in experimental autoimmune encephalomyelitis. J Neurosci 31:11914-11928. CrossRef Medline

Guo F, Maeda Y, Ko EM, Delgado M, Horiuchi M, Soulika A, Miers L, Burns T, Itoh T, Shen H, Lee E, Sohn J, Pleasure D (2012) Disruption of NMDA receptors in oligodendroglial lineage cells does not alter their susceptibility to experimental autoimmune encephalomyelitis or their normal development. J Neurosci 32:639-645. CrossRef Medline

Hammond E, Lang J, Maeda Y, Pleasure D, Angus-Hill M, Xu J, Horiuchi M, Deng W, Guo F (2015) The wnt effector transcription factor 7-like 2 positively regulates oligodendrocyte differentiation in a manner independent of Wnt/beta-catenin signaling. J Neurosci 35:5007-5022. CrossRef Medline

He D, Marie C, Zhao C, Kim B, Wang J, Deng Y, Clavairoly A, Frah M, Wang H, He X, Hmidan H, Jones BV, Witte D, Zalc B, Zhou X, Choo DI, Martin DM, Parras C, Lu QR (2016) Chd7 cooperates with Sox10 and regulates the onset of CNS myelination and remyelination. Nat Neurosci 19:678689. CrossRef Medline

He L, Lu QR (2013) Coordinated control of oligodendrocyte development by extrinsic and intrinsic signaling cues. Neurosci Bull 29:129-143. CrossRef Medline

Heinrich C, Bergami M, Gascon S, Lepier A, Vigano F, Dimou L, Sutor B, Berninger B, Gotz M (2014) Sox2-mediated conversion of NG2 glia into induced neurons in the injured adult cerebral cortex. Stem Cell Reports 3:1000-1014. CrossRef Medline

Hoffmann SA, Hos D, Küspert M, Lang RA, Lovell-Badge R, Wegner M, Reiprich S (2014) Stem cell factor Sox2 and its close relative Sox3 have differentiation functions in oligodendrocytes. Development 141:39-50. CrossRef Medline

Jones MV, Nguyen TT, Deboy CA, Griffin JW, Whartenby KA, Kerr DA, Calabresi PA (2008) Behavioral and pathological outcomes in MOG 35-55 experimental autoimmune encephalomyelitis. J Neuroimmunol 199:83-93. CrossRef Medline

Kondo T, Raff M (2004) Chromatin remodeling and histone modification in the conversion of oligodendrocyte precursors to neural stem cells. Genes Dev 18:2963-2972. CrossRef Medline

Lang J, Maeda Y, Bannerman P, Xu J, Horiuchi M, Pleasure D, Guo F (2013) Adenomatous polyposis coli regulates oligodendroglial development. J Neurosci 33:3113-3130. CrossRef Medline

Lappe-Siefke C, Goebbels S, Gravel M, Nicksch E, Lee J, Braun PE, Griffiths IR, Nave KA (2003) Disruption of Cnp1 uncouples oligodendroglial functions in axonal support and myelination. Nat Genet 33:366-374. CrossRef Medline

Lyssiotis CA, Walker J, Wu C, Kondo T, Schultz PG, Wu X (2007) Inhibition of histone deacetylase activity induces developmental plasticity in oligodendrocyte precursor cells. Proc Natl Acad Sci U S A 104:1498214987. CrossRef Medline

Matsuoka T, Ahlberg PE, Kessaris N, Iannarelli P, Dennehy U, Richardson WD, McMahon AP, Koentges G (2005) Neural crest origins of the neck and shoulder. Nature 436:347-355. CrossRef Medline

Miron VE, Kuhlmann T, Antel JP (2011) Cells of the oligodendroglial lineage, myelination, and remyelination. Biochim Biophys Acta 1812:184193. CrossRef Medline

Moyon S, Huynh JL, Dutta D, Zhang F, Ma D, Yoo S, Lawrence R, Wegner M, John GR, Emery B, Lubetzki C, Franklin RJ, Fan G, Zhu J, Dupree JL, Casaccia
P (2016) Functional characterization of DNA methylation in the oligodendrocyte lineage. Cell Rep 13:S2211-1247(16)30331-X. CrossRef Medline

Niu W, Zang T, Zou Y, Fang S, Smith DK, Bachoo R, Zhang CL (2013) In vivo reprogramming of astrocytes to neuroblasts in the adult brain. Nat Cell Biol 15:1164-1175. CrossRef Medline

Oliver-De La Cruz J, Carrión-Navarro J, García-Romero N, Gutiérrez-Martín A, Lázaro-Ibáñez E, Escobedo-Lucea C, Perona R, Belda-Iniesta C, Ayuso-Sacido A (2014) $\mathrm{SOX}^{+}$cell population from normal human brain white matter is able to generate mature oligodendrocytes. PLoS One 9:e99253. CrossRef Medline

Pedre X, Mastronardi F, Bruck W, López-Rodas G, Kuhlmann T, Casaccia P (2011) Changed histone acetylation patterns in normal-appearing white matter and early multiple sclerosis lesions. J Neurosci 31:3435-3445. CrossRef Medline

Shen S, Sandoval J, Swiss VA, Li J, Dupree J, Franklin RJ, Casaccia-Bonnefil P (2008) Age-dependent epigenetic control of differentiation inhibitors is critical for remyelination efficiency. Nat Neurosci 11:1024-1034. CrossRef Medline

Sohn J, Selvaraj V, Wakayama K, Orosco L, Lee E, Crawford SE, Guo F, Lang J, Horiuchi M, Zarbalis K, Itoh T, Deng W, Pleasure D (2012) PEDF is a novel oligodendrogenic morphogen acting on the adult SVZ and corpus callosum. J Neurosci 32:12152-12164. CrossRef Medline

Sohn J, Bannerman P, Guo F, Burns T, Miers L, Croteau C, Singhal NK, McDonough JA, Pleasure D (2017) Suppressing N-acetyl-l-aspartate synthesis prevents loss of neurons in a murine model of canavan leukodystrophy. J Neurosci 37:413-421. CrossRef Medline

Steelman AJ, Thompson JP, Li J (2012) Demyelination and remyelination in anatomically distinct regions of the corpus callosum following cuprizone intoxication. Neurosci Res 72:32-42. CrossRef Medline

Tripathi RB, Rivers LE, Young KM, Jamen F, Richardson WD (2010) NG2 glia generate new oligodendrocytes but few astrocytes in a murine experimental autoimmune encephalomyelitis model of demyelinating disease. J Neurosci 30:16383-16390. CrossRef Medline

Wheeler NA, Fuss B (2016) Extracellular cues influencing oligodendrocyte differentiation and (re)myelination. Exp Neurol 283:512-530. CrossRef Medline

Yeung MS, Zdunek S, Bergmann O, Bernard S, Salehpour M, Alkass K, Perl S, Tisdale J, Possnert G, Brundin L, Druid H, Frisén J (2014) Dynamics of oligodendrocyte generation and myelination in the human brain. Cell 159:766-774. CrossRef Medline

Zhang S, Cui W (2014) Sox2, a key factor in the regulation of pluripotency and neural differentiation. World J Stem Cells 6:305-311. CrossRef Medline

Zhang SC, Ge B, Duncan ID (1999) Adult brain retains the potential to generate oligodendroglial progenitors with extensive myelination capacity. Proc Natl Acad Sci U S A 96:4089-4094. CrossRef Medline

Zhao C, Ma D, Zawadzka M, Fancy SP, Elis-Williams L, Bouvier G, Stockley JH, de Castro GM, Wang B, Jacobs S, Casaccia P, Franklin RJ (2015) Sox2 sustains recruitment of oligodendrocyte progenitor cells following CNS demyelination and primes them for differentiation during remyelination. J Neurosci 35:11482-11499. CrossRef Medline

Zhao C, Deng Y, Liu L, Yu K, Zhang L, Wang H, He X, Wang J, Lu C, Wu LN, Weng Q, Mao M, Li J, van Es JH, Xin M, Parry L, Goldman SA, Clevers H, Lu QR (2016) Dual regulatory switch through interactions of Tcf7l2/ Tcf4 with stage-specific partners propels oligodendroglial maturation. Nat Commun 7:10883. CrossRef Medline 